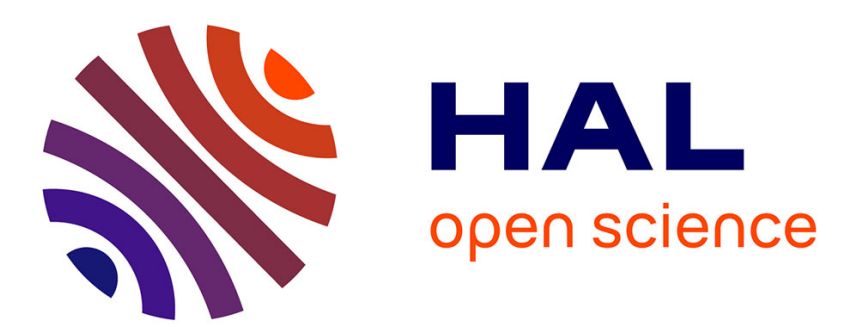

\title{
L'assimilation scolaire des enfants issus de l'immigration et son interprétation: un examen sur données françaises
} Louis-André Vallet

\section{To cite this version:}

Louis-André Vallet. L'assimilation scolaire des enfants issus de l'immigration et son interprétation: un examen sur données françaises. Revue Française de Pédagogie, 1996, 117, pp.7-27. 10.3406/rfp.1996.1182 . hal-03455525

\section{HAL Id: hal-03455525 \\ https://hal.science/hal-03455525}

Submitted on 29 Nov 2021

HAL is a multi-disciplinary open access archive for the deposit and dissemination of scientific research documents, whether they are published or not. The documents may come from teaching and research institutions in France or abroad, or from public or private research centers.
L'archive ouverte pluridisciplinaire HAL, est destinée au dépôt et à la diffusion de documents scientifiques de niveau recherche, publiés ou non, émanant des établissements d'enseignement et de recherche français ou étrangers, des laboratoires publics ou privés. 


\section{L'assimilation scolaire des enfants issus de l'immigration et son interprétation : un examen sur données françaises \\ M. Louis-André Vallet}

\section{Citer ce document / Cite this document :}

Vallet Louis-André. L'assimilation scolaire des enfants issus de l'immigration et son interprétation : un examen sur données françaises. In: Revue française de pédagogie, volume 117, 1996. L'école et la question de l'immigration. pp. 7-27;

doi : https://doi.org/10.3406/rfp.1996.1182

https://www.persee.fr/doc/rfp_0556-7807_1996_num_117_1_1182

Fichier pdf généré le 24/12/2018 


\title{
Résumé
}

Dans cet article, nous désignons par assimilation scolaire des minorités issues de l'immigration le processus temporel par lequel, avec l'avancement dans la scolarité, les parcours des enfants de ces minorités s'améliorent et convergent en moyenne vers ceux des autres élèves. Des données longitudinales sont nécessaires pour éprouver l'existence d'un tel processus. Nous le mettons en évidence sur le panel national 1989 d'élèves du second degré (Ministère de l'Éducation nationale, DEP) en comparant la réussite des parcours scolaires à l'école élémentaire et au collège. L'assimilation scolaire des minorités issues de l'immigration peut trouver sa source dans un processus cognitif de rattrapage des performances et/ou dans des facteurs socio-psychologiques liés à des aspirations spécifiques. Elle pourrait aussi être affectée par le comportement des enseignants à l'égard des élèves de ces minorités et par l'environnement contextuel de leur scolarisation. Nous présentons des analyses empiriques qui tentent d'approcher ces différents effets. Elles soulignent surtout le rôle des facteurs socio-psychologiques : les familles immigrées ont, ceteris paribus, des attentes plus fortes en matière d'études longues et expriment des souhaits d'orientation plus ambitieux pour leurs enfants. Ce résultat suggère que, dans la société française, le système éducatif apparaît aux familles immigrées comme une voie importante de mobilité sociale.

\begin{abstract}
In this paper, school assimilation of minorities stemming from immigration denotes the temporal process by which, in the course of schooling, academic careers of these minorities improve and converge on average on those of other pupils. Longitudinal data are needed to test the existence of such a process. We reveal it using the 1989 French National Education Longitudinal Study (Ministry of Education, DEP) which compares academic careers in elementary school and lower secondary school. School assimilation of minorities stemming from immigration could be due to a cognitive process where performances are catching up and/or to socio- psychological factors related to specific aspirations. It could also be modified by the behaviour of teachers towards pupils belonging to minorities and by the contextual environment of their schooling. We present empirical analyses which try to disentangle these different effects. Above all, they underline the part of socio-psychological factors : ceteris paribus, immigrant families have stronger aspirations towards long studies and express more ambitious orientation plans for their children. This result suggests that, in the French society, the educational system appears to immigrant families as an important vehicle for social mobility.
\end{abstract}




\title{
L'assimilation scolaire des enfants issus de l'immigration et son interprétation : un examen sur données françaises *
}

\author{
Lovis-André Vallet
}

Dans cet article, nous désignons par assimilation scolaire des minorités issues de l'immigration le processus temporel par lequel, avec l'avancement dans la scolarité, les parcours des enfants de ces minorités s'améliorent et convergent en moyenne vers ceux des autres élèves. Des données longitudinales sont nécessaires pour éprouver l'existence d'un tel processus. Nous le mettons en évidence sur le panel national 1989 d'élèves du second degré (Ministère de l'Éducation nationale, DEP) en comparant la réussite des parcours scolaires à l'école élémentaire et au collège. L'assimilation scolaire des minorités issues de l'immigration peut trouver sa source dans un processus cognitif de rattrapage des performances et/ou dans des facteurs socio-psychologiques liés à des aspirations spécifiques. Elle pourrait aussi être affectée par le comportement des enseignants à l'égard des élèves de ces minorités et par l'environnement contextuel de leur scolarisation. Nous présentons des analyses empiriques qui tentent d'approcher ces différents effets. Elles soulignent surtout le rôle des facteurs socio-psychologiques: les familles immigrées ont, ceteris paribus, des attentes plus fortes en matière d'études longues et expriment des souhaits d'orientation plus ambitieux pour leurs enfants. Ce résultat suggère que, dans la société française, le système éducatif apparaît aux familles immigrées comme une voie importante de mobilité sociale.

'analyse sociologique des migrations désigne Lpar assimilation le processus temporel par lequel les traits spécifiques des minorités issues de l'immigration se réduisent et leurs comportements et attributs convergent vers ceux qui caractérisent les membres de la société d'accueil (Alba et Nee, 1996 ; Tribalat, 1996). Sur le plan socioéconomique par exemple, la théorie de l'assimilation conduit à prédire la résorption progressive des inégalités de niveau d'éducation, de position professionnelle et de revenu qui séparent les minorités de la majorité. Les tests empiriques de

\footnotetext{
- Cet article utilise les résultats empiriques établis dans le cadre d'une convention de recherche de la Direction de l'Évaluation et de la Prospective (Ministère de l'Éducation nationale, Paris); voir, pour la publication principale de cette recherche, Vallet et Caille (1996a).
} 
cette théorie mettent souvent en jeu une conception longue du temps de l'assimilation, celui de la succession des générations. Ils consistent alors à comparer, à un instant donné et pour les mêmes critères, des individus qui appartiennent aux première, deuxième et, éventuellement, troisième générations de l'immigration, c'est-à-dire respectivement des immigrés et des enfants (voire des petits-enfants) nés dans la société d'accueil de parents (ou de grands-parents) immigrés (Neidert et Farley, 1985). D'autres tests de l'hypothèse de l'assimilation ne portent que sur la seule génération des immigrés et distinguent alors ceux-ci selon l'ancienneté de leur arrivée dans la société d'accueil.

Toutes ces approches ont en commun d'utiliser des données synchroniques (ou transversales) recueillies sur des populations distinctes pour lesquelles il s'agit d'examiner à quel point elles sont parvenues à des degrés différents d'assimilation. Dans cet article, nous envisagerons la théorie de l'assimilation dans une perspective distincte, individuelle et diachronique (ou longitudinale). Elle consiste à observer comment se transforment au cours du temps les comportements et attributs des mêmes individus. C'est alors une conception plus courte du temps de l'assimilation qui est privilégiée.

\section{L'HYPOTHĖSE DE L'ASSIMILATION SCOLAIRE}

Nous entendrons donc par assimilation scolaire des minorités issues de l'immigration le processus par lequel, avec l'avancement dans la scolarité, les parcours des enfants de ces minorités s'améliorent et convergent en moyenne vers ceux dont font preuve les autres élèves. Supposons ainsi un échantillon d'enfants pour lesquels le déroulement des études à l'école élémentaire, puis au collège a été observé et retenons par exemple l'absence de redoublement comme indicateur de réussite du parcours. On sera fondé à parler d'assimilation scolaire dans le cas où le handicap des minorités issues de l'immigration se réduit dans le temps, c'est-à-dire lorsque l'écart entre ces élèves et leurs condisciples pour le taux de scolarités secondaires sans redoublement s'avère moins important que l'écart correspondant mesuré à propos des scolarités primaires des mêmes élèves.
C'est en premier lieu à propos des enfants immigrés, c'est-à-dire nés étrangers dans un pays étranger, que l'hypothèse de l'assimilation scolaire peut être formulée. Dans le cadre de notre exemple, leur migration et leur entrée dans la société d'accueil constituent par définition des événements plus anciens lors de l'observation de la scolarité au collège qu'ils ne le sont à l'instant où l'on mesure la réussite du parcours à l'école. S'agissant des enfants immigrés, l'hypothèse de l'assimilation scolaire exprime alors l'effet positif de l'exposition à la société d'accueil et à son institution scolaire, l'adaptation des enfants augmentant avec la durée de cette exposition. L'hypothèse de l'assimilation scolaire peut aussi être étendue aux enfants nés dans la société d'accueil de parent(s) immigré(s): s'ils grandissent dans une famille où l'empreinte de la culture et de la langue du pays d'origine reste forte, la fréquentation prolongée de l'école de la société d'accueil doit sans doute être considérée comme un facteur important du processus de leur acculturation.

La formulation de l'hypothèse d'assimilation scolaire découle partiellement de résultats empiriques fournis par le panel national d'élèves recruté dans les classes de $6^{e}$ en 1972, 1973 et 1974 (1). On y observait notamment qu'entre le premier degré et le second degré et par rapport aux élèves français de la même catégorie sociale, la situation scolaire des jeunes étrangers nés en France s'inversait. La réussite à l'école des enfants nés en France dont le père, étranger, était ouvrier non qualifié apparaissait inférieure à celle de leurs condisciples français du même milieu : $31,6 \%$ des premiers et $36,9 \%$ des seconds n'y avaient pas redoublé ; la tendance était identique parmi les enfants d'ouvrier qualifié ; enfin, dans ces deux milieux sociaux, le handicap de carrière scolaire était nettement plus lourd pour les enfants étrangers nés à l'étranger. Les parcours scolaires dans le second degré se présentaient cependant sous un jour différent. Parmi les entrants en $6^{\mathrm{e}}$ (normale ou allégée) des catégories sociales défavorisées, le taux d'admission en terminale ne différait guère chez les élèves français et leurs condisciples étrangers. Calculé sur les seuls enfants d'ouvrier non qualifié, il s'agissait de $16,2 \%$ des jeunes Français, 18,1\% des étrangers nés en France et $11,1 \%$ des étrangers nés à l'étranger. Ces proportions valaient respectivement $24,7 \%, 28,9 \%$ et $23,3 \%$ parmi les enfants d'ouvrier qualifié. Les cheminements des élèves nés en France d'un père étranger et ouvrier 
étaient devenus, dans l'enseignement secondaire, plus favorables que ceux de leurs condisciples français d'origine sociale identique. Ce n'était pas le cas dans l'enseignement primaire.

L'hypothèse d'assimilation scolaire conduit donc à prédire la réduction progressive des écarts bruts de réussite entre les enfants issus de l'immigration et les autres. Ces écarts ne constituent pas, au sens strict, une mesure de l'influence de l'appartenance aux minorités. D'une manière générale, ils dépendent de toutes les caractéristiques sociodémographiques qui affectent la réussite scolaire - appartenance sociale, niveau d'éducation des parents, taille des fratries notamment - tout en différenciant en moyenne les minorités issues de l'immigration des autres familles. Des modèles statistiques d'analyse multivariée permettent de démêler l'écheveau des effets et d'isoler ainsi l'influence, sur la réussite à l'école, propre à l'appartenance aux minorités. On peut donc dériver de l'hypothèse d'assimilation scolaire une seconde prédiction. Les paramètres des modèles statistiques qui expriment l'effet de l'appartenance aux minorités doivent se transformer avec l'avancement dans la scolarité, la tendance temporelle étant celle de la réduction d'un effet négatif ou de l'accentuation d'un effet positif. Par contraste, les paramètres qui expriment l'influence des autres caractéristiques socio-démographiques doivent faire preuve d'une plus grande stabilité.

\section{LES DÉTERMINANTS DE L'ASSIMILATION SCOLAIRE}

Si elle existe, l'assimilation scolaire des minorités issues de l'immigration pourrait trouver sa source dans un processus cognitif de rattrapage des performances, c'est-à-dire dans le fait que, sur le plan des acquis mesurés au moyen d'épreuves standardisées, les élèves des minorités progressent en moyenne plus fortement que les autres élèves de caractéristiques analogues. Dans la littérature disponible, plusieurs études tendent à accréditer ce résultat. Elles font appel à des modèles d'analyse de covariance pour "expliquer" la variation d'un niveau de performance final en fonction d'une batterie de variables qui inclut une mesure initiale de la même compétence. Le coefficient de régression estimé pour une sous-population d'élèves particulière s'interprète alors comme le fait que ceux-ci ont, en moyenne, plus, autant ou moins progressé durant la période considérée que les autres élèves qui possèdent par ailleurs les mêmes caractéristiques.

En Angleterre, dans une étude longitudinale auprès de vingt écoles secondaires multiraciales, Smith et Tomlinson (1989) ont ainsi observé de manière consistante qu'entre les âges de 13 et 16 ans les élèves des minorités avaient davantage progressé en anglais et en mathématiques que leurs condisciples des mêmes milieux sociaux. En France, un résultat analogue a été obtenu sur un échantillon de près de 3000 élèves observé en début et en fin de classe de CE2 (Bressoux, 1994) comme dans deux études traitant du déroulement des scolarités au cours des deux premières années du collège (Ernst et Radica, 1994 ; Meuret, 1994). Mingat (1991) ne conclut en revanche à une meilleure progression, durant l'année de cours préparatoire, que pour les enfants étrangers nés à l'étranger et obtient un résultat inverse pour les enfants étrangers nés en France. Enfin, Serra et Thaurel-Richard (1994) ont observé que la nationalité de l'élève n'engendrait pas de différence significative dans les acquisitions réalisées durant la seconde année du cours élémentaire.

Un autre déterminant de l'assimilation scolaire des élèves issus de l'immigration pourrait tenir au fait que ces enfants et leurs familles développent, à l'égard du système scolaire de la société d'accueil, des attentes et des aspirations plus fortes que celles des autres membres des mêmes milieux sociaux. Si le désir d'une vie meilleure et d'une ascension sociale a constitué souvent un mobile important de la décision d'émigration des familles, beaucoup de celles-ci occupent néanmoins, dans le pays d'immigration, des positions sociales inférieures. Les familles immigrées pourraient alors percevoir l'investissement dans le système scolaire comme la voie principale de mobilité ascendante qui leur est accessible. Par rapport aux autres familles dotées des mêmes ressources matérielles et culturelles - liées notamment à leur position sociale et à leur niveau d'éducation -, elles souhaiteraient donc plus vivement que leurs enfants acquièrent une qualification scolaire élevée.

Certaines recherches étrangères soulignent l'existence de tels facteurs socio-psychologiques. Dans une exploitation de la National Education Longitudinal Study, c'est-à-dire du panel américain qui a observé, à partir de 1988, un échantillon 
de 26000 élèves scolarisés au niveau de la classe de $4^{e}$, Muller et Kerbow (1993) présentent une analyse où la proportion de parents qui envisagent que leur enfant obtienne un diplôme universitaire figure dans un graphique qui tient compte à la fois de leur niveau d'études et de la variable race/ethnicité. Sans aucune exception et pour chaque diplôme parental, le point du graphique associé aux Américains de race blanche est situé en dessous des trois autres qui concernent les minorités asiatique, hispanique et noire. Muller et Kerbow interprètent ce résultat comme le signe que les parents des minorités sont plus sensibles que les autres aux bénéfices sociaux que l'éducation apporte. Dans une étude longitudinale australienne, Clifton et al. (1991) ont observé que les élèves d'origine grecque et italienne obtenaient à l'âge de 14 ans des performances en anglais et en arithmétique inférieures à celles des autres élèves de mêmes caractéristiques, mais qu'ils étaient plus nombreux à parvenir au terme des études secondaires. Les analyses statistiques des auteurs mettent en évidence le rôle des facteurs socio-psychologiques: les élèves des minorités trouvent dans leur environnement - amis, parents et professeurs - des encouragements plus vifs concernant leurs études et développent aussi une conception plus positive de leur valeur scolaire.

En France également, dans sa conclusion d'une étude de deux années auprès d'une centaine de collèges, Grisay (1993) souligne que les élèves issus de l'immigration paraissent en moyenne mieux disposés envers l'école que les jeunes Français de même catégorie socioprofessionnelle, qu'ils sont plus désireux de "bien faire " et de se conformer aux attentes de leurs professeurs. Des observations voisines ont été faites en Angleterre (Smith et Tomlinson, 1989). En revanche, DuruBellat et Jarousse (1996) n'ont pas observé, dans notre pays, de différence significative entre familles étrangères et familles françaises de niveau d'instruction et catégorie sociale identiques pour ce qui est de l'espoir que leur enfant parvienne au baccalauréat.

La progression plus forte des performances scolaires et le rôle des aspirations familiales constituent deux hypothèses complémentaires susceptibles de rendre compte du processus d'assimilation scolaire des élèves issus de l'immigration. Leurs cheminements pourraient dépendre aussi des évaluations portées par les professeurs aux paliers du système éducatif où des décisions d'orientation interviennent si l'appartenance aux minorités est un élément pris en compte dans la formation de ces évaluations (Clifton et al., 1986). Enfin, la scolarisation des enfants issus de l'immigration s'effectue souvent dans des établissements où ces élèves sont nombreux et où la concentration des handicaps sociaux est forte. L'environnement contextuel de la scolarisation des enfants des minorités pourrait ainsi affecter leurs cheminements. Des analyses multivariées détaillées sont nécessaires pour apprécier les effets de ces différents facteurs.

\section{DONNÉES FRANÇAISES DISPONIBLES}

Pour éprouver l'hypothèse de l'assimilation scolaire, nous tirerons profit des données du panel national 1989 d'élèves du second degré (Ministère de l'Éducation nationale, DEP). Plus précisément, notre échantillon comprend l'ensemble des enfants nés le 5 d'un mois qui, en septembre 1989 , entraient au collège en classe de $6^{\mathrm{e}}$ dans un établissement public ou privé de France métropolitaine et dont la famille a répondu à une enquête complémentaire au printemps 1991. Un test de l'hypothèse de l'assimilation peut être conduit en étudiant la réussite des parcours scolaires que ces élèves ont effectués dans l'école élémentaire française avant septembre 1989, puis au collège après cette date.

La mesure de la réussite à l'école élémentaire utilise l'information rétrospective recueillie, pour chaque élève de l'échantillon, d'une part à l'automne 1989 auprès du collège fréquenté, d'autre part au printemps 1991 auprès de la famille. Nous considérons que l'élève a redoublé au cours des années passées dans l'école élémentaire française lorsque le collège et/ou la famille a fourni cette information pour l'un au moins des cinq niveaux de classe en question. L'indicateur de réussite retenu consiste alors en l'absence de redoublement dans l'école française. L'information est disponible pour 18657 élèves, parmi lesquels $74,5 \%$ ont connu cette situation de succès.

La mesure de la réussite au collège utilise l'information collectée auprès de l'établissement scolaire pendant les quatre années qui ont suivi l'entrée en classe de $6^{\mathrm{e}}$, c'est-à-dire jusqu'en juin 1993. Au cours de cette période, l'attrition du panel a été faible ; notre échantillon comprend ici 18538 élèves. 
Nous retenons un indicateur de réussite qui combine absence de redoublement au collège et orientation vers des études longues conduisant au baccalauréat. II est défini ainsi : avoir reçu, quatre ans après l'entrée au collège, une proposition d'orientation en classe de seconde générale ou technologique ; $47,1 \%$ des élèves considérés ont connu cette situation de succès (2).

L'information sur les caractéristiques sociodémographiques des élèves et de leurs familles a été recueillie auprès de celles-ci et/ou de l'établissement de scolarisation. Neuf variables nous permettront d'approcher la situation familiale et sociale des élèves, c'est-à-dire à la fois les ressources et les contraintes objectives qu'ils trouvent dans leur environnement : la catégorie socioprofessionnelle de la personne de référence de la famille, le diplôme le plus élevé du père, le diplôme le plus élevé de la mère, le fait que celleci est inactive ou bien exerce (ou recherche) une activité professionnelle, le nombre total d'enfants, le sexe de l'élève, son rang de naissance, le fait qu'il a (ou n'a pas) de frère ou sœur plus âgé(e) scolarisé(e) en lycée d'enseignement général et technologique ou dans l'enseignement supérieur, la structure de la famille. Cette dernière variable distingue les élèves qui vivent avec leurs deux parents (ou dans une famille recomposée), ceux qui appartiennent à une famille monoparentale, ceux enfin qui vivent avec un autre membre de leur parenté ou connaissent une situation particulière (orphelins en foyer notamment).

Isoler rigoureusement la population des enfants immigrés - c'est-à-dire nés étrangers à l'étranger - ou nés en France de parent(s) immigré(s) nécessite de connaître, pour les élèves de l'échantillon, le pays où ils sont nés, leur nationalité à la naissance et des informations similaires concernant leurs parents. Certaines de ces données ne sont pas disponibles dans le panel d'élèves. II est possible en revanche d'approcher cette population à l'aide de huit critères tous liés, d'un point de vue statistique, à la migration de l'enfant ou de ses parents. C'est en ce sens que, dans la suite, nous utilisons l'expression d'appartenance à la population étrangère ou issue de l'immigration.

Nationalité de l'élève en deux postes (Français/ étranger). Elle a été recueillie par les collèges lors de la mise en place du panel. Pour 7,8\% des élèves considérés, une nationalité étrangère a été indiquée (3).
Nationalité de l'élève en sept postes. Le recueil de l'appartenance nationale utilisait une nomenclature plus détaillée. Six nationalités (ou groupes de nationalités) étrangères ont donc été distinguées: Maghreb (49,9\% des élèves étrangers), autre pays d'Afrique $(4,5 \%)$, Europe du Sud $(23,5 \%)$, Asie du Sud-Est $(6,5 \%)$, Turquie $(8,1 \%)$, autre pays $(7,5 \%)$.

Lieu de naissance de l'élève. À partir de l'information fournie par l'établissement scolaire, les naissances en France métropolitaine, dans un département ou un territoire d'outre-mer, ou dans un pays étranger ont été distinguées ; $0,2 \%$ des élèves considérés sont nés dans les Dom-Tom et $4,5 \%$ à l'étranger.

Nombre d'années scolaires hors de France. Fournie par les principaux de collège lors de l'entrée en sixième, cette information concerne la scolarité primaire et est affectée d'un fort taux de non-réponse $(37,5 \%)$. Il a justifié la création d'une modalité "inconnu ». Trois autres situations ont été distinguées : aucune année scolaire hors de France, une ou deux années, trois années ou davantage. Les élèves ayant passé au moins une année scolaire hors de France représentent 2,1\% des collégiens pour lesquels l'information est disponible.

Ancienneté en France des parents. Dans l'enquête auprès des familles, cette question était posée séparément pour le père et la mère et l'on a pris en compte la durée de présence la plus élevée. Quatre modalités ont été retenues: I'un des parents au moins a toujours vécu en France ( $84,3 \%$ des élèves considérés); aucun des parents n'a toujours vécu en France, mais l'un au moins y réside depuis plus de 20 ans $(7,4 \%)$; aucun des parents n'est en France depuis plus de 20 ans, mais l'un au moins y habite depuis 5 ans $(5,6 \%)$; les parents sont en France depuis moins de 5 ans $(0,4 \%)$. Une modalité "inconnu " a été ajoutée en cas de non-réponse à la question ou pour les élèves ayant perdu leurs parents $(2,4 \%)$.

Langue parlée à la maison. Il s'agit de la réponse (oui/non) à la question - posée dans l'enquête auprès des familles - "Parlez-vous régulièrement une autre langue que le français avec vos enfants ?"; $13,8 \%$ des parents ont répondu par l'affirmative.

Groupe d'appartenance de l'élève. Cette variable synthétique a été obtenue en combinant la natio- 
nalité en deux postes, le lieu de naissance en deux modalités (France métropolitaine ou autre) et la réponse à la question sur la langue. Huit groupes de collégiens sont ainsi définis, des Français nés en métropole à qui leurs parents ne parlent pas autre chose que le français $(83,9 \%)$ aux élèves de nationalité étrangère, nés hors de France métropolitaine à qui leurs parents parlent régulièrement une autre langue que le français $(2,8 \%)$. Deux autres groupes assez nombreux sont formés des Français nés dans l'hexagone à qui leurs parents parlent régulièrement une langue étrangère $(6,8 \%)$ et des étrangers de lieu de naissance et de situation linguistique analogues $(3,8 \%)$.

Nombre d'attributs étrangers. La possession d'un " attribut étranger " est définie comme le fait de présenter l'une des cinq caractéristiques qui suivent: être de nationalité étrangère, être né hors de France métropolitaine, avoir passé au moins une année scolaire hors de France, n'avoir aucun parent ayant toujours vécu en France, avoir des parents qui parlent régulièrement une autre langue que le français. Au total, $19,7 \%$ des collégiens considérés présentent au moins un attribut étranger : $8,7 \%$ en comptent un, 3,8 \% deux, $4,4 \%$ trois et $2,7 \%$ quatre ou cinq.

\section{L'HYPOTHĖSE DE L'ASSIMILATION SCOLAIRE À L'ÉPREUVE DES DONNÉES FRANÇAISES}

\section{De l'école élémentaire au collège, réduction des écarts bruts de réussite scolaire}

Pour chaque variable d'appartenance à la population étrangère ou issue de l'immigration, le tableau 1 présente le taux de réussite des parcours (école élémentaire et collège) pour le groupe d'élèves le plus nombreux, puis les écarts des autres groupes à celui-ci. Ainsi, 76,3 \% des entrants en $6^{\mathrm{e}}$ de nationalité française n'ont pas redoublé à l'école élémentaire ; ils sont $48,3 \%$ à connaître cette situation au collège et à être orientés en seconde générale ou technologique. Parmi les élèves étrangers, les mêmes taux de réussite des parcours sont inférieurs respectivement de 22,0 points et 15,6 points. Mesuré par la différence de proportions, l'écart de réussite entre Français et étrangers se réduit donc avec l'avancement dans la scolarité. Préciser l'appartenance nationale des enfants étrangers ne modifie pas ce résultat, à l'exception des élèves asiatiques: dès l'école élémentaire, le taux de réussite de leur parcours était assez proche de celui des jeunes Français et l'écart ne se réduit pas ultérieurement.

Approcher la population des enfants issus de l'immigration par le lieu de naissance, le nombre d'années scolaires hors de France, l'ancienneté en France des parents, la langue parlée à la maison, le groupe d'appartenance de l'élève ou le nombre d'attributs étrangers conduit à une conclusion analogue. Conformément à l'hypothèse de l'assimilation scolaire, l'écart au groupe de référence - ou groupe d'élèves le plus nombreux - est généralement plus faible dans le second degré que dans le premier degré. En particulier, la réduction des écarts de réussite est nette pour les enfants nés à l'étranger, ceux qui ont passé au moins trois années scolaires hors de France, les élèves dont la famille réside en France depuis 5 à 20 ans, les enfants de nationalité étrangère nés hors de métropole et ceux qui comptent quatre ou cinq attributs étrangers (4).

Pour chaque variable d'appartenance, le tableau 2 récapitule l'évolution temporelle de l'écart de réussite entre les groupes d'élèves qui étaient initialement les plus distants. Sans aucune exception, ces écarts se réduisent au cours du temps. La différence de proportions ne constitue toutefois que l'une des mesures possibles de l'inégalité. On peut notamment lui préférer un indicateur plus complexe connu sous le nom de odds ratio ou rapport des chances relatives (Vallet, 1988). Que, pour la réussite du parcours à l'école élémentaire, le odds ratio entre étrangers et Français vaille 2,7 peut alors s'interpréter ainsi : les chances de réussir plutôt que d'échouer dans le premier degré ont été 2,7 fois plus faibles pour les jeunes étrangers que pour les enfants français. Cette valeur se réduit à 1,9 dans le second degré. Mesurée par le odds ratio, l'inégalité de réussite entre étrangers et Français diminue donc avec l'avancement dans les études et les autres critères utilisés pour approcher la population des élèves issus de l'immigration livrent la même conclusion.

\section{De l'école élémentaire au collège, transformation de l'effet d'appartenance aux minorités}

Pour distinguer, dans les écarts bruts de réussite scolaire, la part liée spécifiquement à l'appartenance aux minorités, nous mettons en œuvre 
Tableau 1 - Écarts de réussite du parcours scolaire.

Comparaison entre école élémentaire et collège

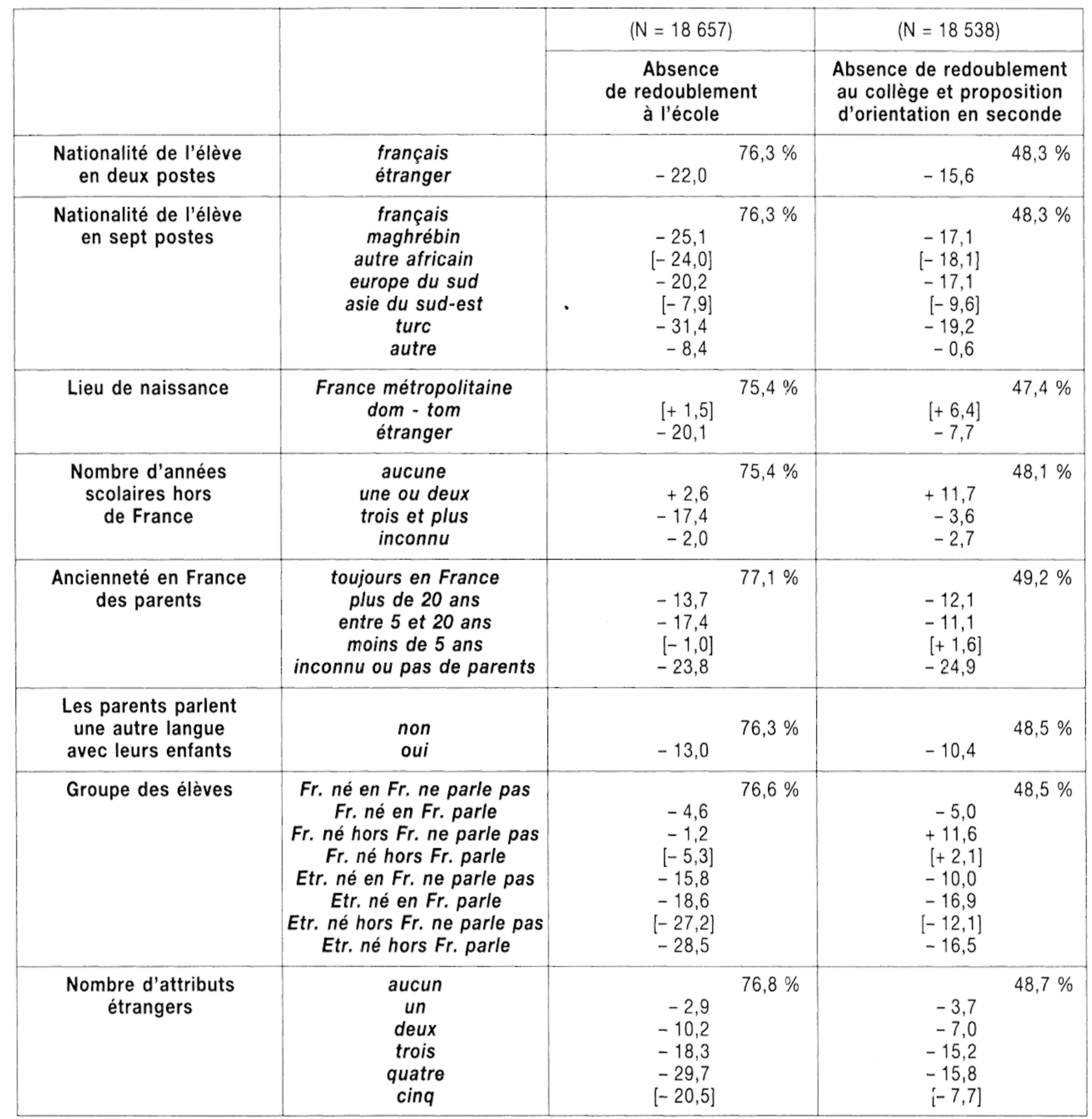

Lecture : $76,3 \%$ des élèves français ont réussi leur scolarité à l'école élémentaire et $48,3 \%$ au collège. Pour les élèves étrangers, ces proportions sont inférieures de 22,0 points (soit $54,3 \%$ à l'école) et 15,6 points (soit $32,7 \%$ au collège). L'écart entre Français et étrangers se réduit donc avec l'avancement dans la scolarité.

Les valeurs entre crochets font référence à des effectifs inférieurs à 100 et doivent donc être interprétées avec prudence.

des modèles de régression logistique. Ils analysent l'effet, sur le succès du parcours à l'école ou au collège, de neuf caractéristiques socio-démographiques qui appréhendent la situation familiale et sociale des élèves (voir plus haut). À ces der- nières est ajoutée, tour à tour et de manière indépendante, chacune des variables d'appartenance à la population étrangère ou issue de l'immigration. En procédant ainsi, nous évaluons donc l'influence spécifique de la nationalité (ou du lieu de 
Tableau 2 - Écarts maximaux de réussite du parcours scolaire (différence de proportions et odds ratio). Comparaison entre école élémentaire et collège

\begin{tabular}{|c|c|c|}
\hline & $\begin{array}{l}\text { Inégalité de réussite } \\
\text { de la scolarité } \\
\text { à l'école élémentaire }\end{array}$ & $\begin{array}{l}\text { Inégalité de réussite } \\
\text { de la scolarité } \\
\text { au collège }\end{array}$ \\
\hline Entre les élèves étrangers et les élèves français & 22 pts / 2,7 & $16 \mathrm{pts} / 1,9$ \\
\hline Entre les élèves turcs et les élèves français & $31 \mathrm{pts} / 4,0$ & 19 pts / 2,3 \\
\hline $\begin{array}{l}\text { Entre les élèves nés à l'étranger et ceux } \\
\text { nés en France métropolitaine } \ldots \ldots \ldots \ldots \ldots \ldots \ldots\end{array}$ & 20 pts / 2,5 & $8 \mathrm{pts} / 1,4$ \\
\hline $\begin{array}{l}\text { Entre les élèves qui ont passé plus } \\
\text { de deux années scolaires hors de France } \\
\text { et ceux dont toutes les études primaires } \\
\text { se sont déroulées dans ce pays } \ldots \ldots \ldots \text {. . . }\end{array}$ & 17 pts / 2,2 & $4 \mathrm{pts} / 1,2$ \\
\hline $\begin{array}{l}\text { Entre les élèves dont la famille réside } \\
\text { en France depuis cinq à vingt ans } \\
\text { et ceux dont un parent au moins a toujours vécu } \\
\text { dans ce pays } \ldots \ldots \ldots \ldots \ldots \ldots \ldots \ldots \ldots \ldots \ldots \ldots \ldots \ldots \ldots\end{array}$ & $17 \mathrm{pts} / 2,3$ & $11 \mathrm{pts} / 1,6$ \\
\hline $\begin{array}{l}\text { Entre les élèves à qui leurs parents parlent } \\
\text { régulièrement une autre langue que le français } \\
\text { et ceux pour qui tel n'est pas le cas ........ }\end{array}$ & 13 pts / 1,9 & 10 pts / 1,5 \\
\hline $\begin{array}{l}\text { Entre les élèves étrangers, nés hors de France } \\
\text { métropolitaine à qui leurs parents parlent régulièrement } \\
\text { une autre langue que le français et les élèves français, } \\
\text { nés en France métropolitaine dont les parents } \\
\text { ne s'expriment qu'en français } \ldots \ldots \ldots \ldots \ldots \ldots \ldots \ldots \ldots\end{array}$ & 29 pts / 3,5 & 17 pts / 2,0 \\
\hline $\begin{array}{l}\text { Entre les élèves qui comptent quatre attributs étrangers } \\
\text { et ceux qui n'en ont aucun } \ldots \ldots \ldots \ldots \ldots \ldots \ldots \ldots \ldots\end{array}$ & $30 \mathrm{pts} / 3,7$ & 16 pts / 1,9 \\
\hline
\end{tabular}

Lecture : Mesurée par la différence de proportions ou par le odds ratio (valeur en italiques) et pour les catégories d'élèves qui étaient les plus distantes à l'école élémentaire, l'inégalité de réussite se réduit avec l'avancement dans la scolarité.

naissance, ou encore de la langue parlée à la maison, etc.), à situation familiale et sociale identique. Les coefficients qui expriment ces effets à l'école élémentaire, puis au collège figurent dans le tableau 3.

S'agissant de la réussite du parcours à l'école élémentaire, le coefficient estimé pour les jeunes étrangers (par rapport aux enfants français) est très proche de 0 et n'en diffère pas significativement. Par comparaison à leurs condisciples qui connaissent le même environnement familial, les élèves étrangers n'ont donc pas été plus nombreux à redoubler dans le premier degré. A propos de la scolarité dans le second degré, le même coefficient s'élève en revanche à $+0,30$ et est très significatif. Les carrières scolaires au collège des élèves étrangers ont donc été meilleures que celles de leurs condisciples de mêmes caractéris- tiques (Vallet et Caille, 1995) (5). Nul puis positif, l'effet spécifique associé à la nationalité étrangère varie avec l'avancement dans les études et cette transformation s'effectue dans une direction compatible avec l'hypothèse d'assimilation scolaire.

Un examen plus général du tableau 3 laisse apparaître, entre école et collège, des évolutions de différents types. La réduction d'un effet négatif tout d'abord. Elle apparaît notamment pour les enfants des " autres nationalités étrangères ", ceux qui ont connu plus de deux années scolaires hors de France et les élèves étrangers nés hors de métropole avec qui leurs parents ne parlent qu'en français. L'apparition ou l'accentuation d'un effet positif ensuite. Elle concerne par exemple les enfants maghrébins ou turcs, ceux dont la famille réside en France depuis 5 à 20 ans, les jeunes étrangers nés 
Tableau 3 - Effet de l'appartenance à la population étrangère ou issue de l'immigration. Comparaison entre école élémentaire et collège

\begin{tabular}{|c|c|c|c|c|c|}
\hline & & \multicolumn{2}{|c|}{$(N=18657)$} & \multicolumn{2}{|c|}{$(\mathrm{N}=18538)$} \\
\hline \multirow[t]{2}{*}{$\begin{array}{l}\text { Modalité } \\
\text { de référence }\end{array}$} & \multirow[t]{2}{*}{$\begin{array}{l}\text { Modalité } \\
\text { active }\end{array}$} & \multicolumn{2}{|c|}{$\begin{array}{l}\text { Absence } \\
\text { de redoublement } \\
\text { à l'école }\end{array}$} & \multicolumn{2}{|c|}{$\begin{array}{l}\text { Absence de redoublement } \\
\text { au collège et proposition } \\
\text { d'orientation en seconde }\end{array}$} \\
\hline & & Coefficient & Test & Coefficient & Test \\
\hline $\begin{array}{c}\text { Nationalité de l'élève } \\
\text { en deux postes } \\
\text { français }\end{array}$ & étranger & 0,01 & ns & 0,30 & .001 \\
\hline $\begin{array}{c}\text { Nationalité de l'élève } \\
\text { en sept postes } \\
\text { français }\end{array}$ & $\begin{array}{c}\text { maghrébin } \\
\text { autre africain } \\
\text { europe du sud } \\
\text { asie du sud-est } \\
\text { turc } \\
\text { autre }\end{array}$ & $\begin{array}{r}0,16 \\
-0,30 \\
-0,15 \\
0,63 \\
-0,10 \\
-0,65\end{array}$ & $\begin{array}{l}.10 \\
\mathrm{~ns} \\
\mathrm{~ns} \\
.01 \\
\mathrm{~ns} \\
.01\end{array}$ & $\begin{array}{r}0,50 \\
-0,16 \\
0,14 \\
0,56 \\
0,47 \\
-0,29\end{array}$ & $\begin{array}{l}.001 \\
\text { ns } \\
\text { ns } \\
.02 \\
.05 \\
\text { ns }\end{array}$ \\
\hline $\begin{array}{l}\text { Lieu de naissance } \\
\text { France métropolitaine }\end{array}$ & $\begin{array}{c}\text { dom - tom } \\
\text { étranger }\end{array}$ & $\begin{array}{r}0,25 \\
-0,35\end{array}$ & $\begin{array}{l}\mathrm{ns} \\
.001\end{array}$ & $\begin{array}{l}0,37 \\
0,16\end{array}$ & $\begin{array}{l}\text { ns } \\
.10\end{array}$ \\
\hline $\begin{array}{l}\text { Nombre d'années } \\
\text { scolaires hors } \\
\text { de France } \\
\text { aucune }\end{array}$ & $\begin{array}{l}\text { une ou deux } \\
\text { trois et plus } \\
\text { inconnu }\end{array}$ & $\begin{array}{l}-0,10 \\
-0,82 \\
-0,10\end{array}$ & $\begin{array}{l}\text { ns } \\
.001 \\
.01\end{array}$ & $\begin{array}{r}0,18 \\
-0,15 \\
-0,11\end{array}$ & $\begin{array}{l}\mathrm{ns} \\
\mathrm{ns} \\
.01\end{array}$ \\
\hline $\begin{array}{c}\text { Ancienneté en France } \\
\text { des parents } \\
\text { toujours en France }\end{array}$ & $\begin{array}{c}\text { plus de } 20 \text { ans } \\
\text { entre } 5 \text { et } 20 \text { ans } \\
\text { moins de } 5 \text { ans } \\
\text { inconnu ou pas de parents }\end{array}$ & $\begin{array}{r}0,15 \\
0,05 \\
-0,09 \\
-0,40\end{array}$ & $\begin{array}{l}.05 \\
\mathrm{~ns} \\
\mathrm{~ns} \\
.001\end{array}$ & $\begin{array}{r}0,21 \\
0,30 \\
-0,20 \\
-0,48\end{array}$ & $\begin{array}{l}.01 \\
.001 \\
\mathrm{~ns} \\
.001\end{array}$ \\
\hline $\begin{array}{l}\text { Les parents parlent } \\
\text { une autre langue } \\
\text { avec leurs enfants } \\
\text { non }\end{array}$ & oui & 0,06 & ns & 0,13 & .02 \\
\hline $\begin{array}{c}\text { Groupe des élèves } \\
\text { Français, nés en France } \\
\text { métropolitaine, } \\
\text { les parents ne parlent pas } \\
\text { autre chose } \\
\text { que le français }\end{array}$ & $\begin{array}{l}\text { Fr. né en Fr. parle } \\
\text { Fr. né hors Fr. ne parle pas } \\
\text { Fr. né hors Fr. parle } \\
\text { Etr. né en Fr. ne parle pas } \\
\text { Etr. né en Fr. parle } \\
\text { Etr. né hors Fr. ne parle pas } \\
\text { Etr. né hors Fr. parle }\end{array}$ & $\begin{array}{r}0,07 \\
-0,25 \\
-0,14 \\
0,21 \\
0,22 \\
-0,79 \\
-0,23\end{array}$ & $\begin{array}{l}\text { ns } \\
\text { ns } \\
\text { ns } \\
\text { ns } \\
.02 \\
.01 \\
.05\end{array}$ & $\begin{array}{r}0,03 \\
0,30 \\
0,18 \\
0,54 \\
0,34 \\
-0,09 \\
0,26\end{array}$ & $\begin{array}{l}\mathrm{ns} \\
.10 \\
\mathrm{~ns} \\
.01 \\
.001 \\
\mathrm{~ns} \\
.05\end{array}$ \\
\hline $\begin{array}{c}\text { Nombre d'attributs } \\
\text { étrangers } \\
\text { aucun }\end{array}$ & $\begin{array}{l}\text { un } \\
\text { deux } \\
\text { trois } \\
\text { quatre } \\
\text { cinq }\end{array}$ & $\begin{array}{r}-0,03 \\
0,20 \\
0,15 \\
-0,21 \\
-0,47\end{array}$ & $\begin{array}{l}\text { ns } \\
.05 \\
.10 \\
.10 \\
\text { ns }\end{array}$ & $\begin{array}{r}-0,08 \\
0,34 \\
0,31 \\
0,38 \\
0,09\end{array}$ & $\begin{array}{l}\mathrm{ns} \\
.001 \\
.001 \\
.01 \\
\mathrm{~ns}\end{array}$ \\
\hline
\end{tabular}

Lecture : Chaque variable d'appartenance est introduite de manière indépendante dans une régression logistique qui inclut par ailleurs CSP de la personne de référence de la famille, diplôme le plus élevé du père, diplôme le plus élevé de la mère, activité ou inactivité professionnelle de celle$\mathrm{ci}$, structure de la famille, nombre total d'enfants, présence/absence d'un aîné scolarisé au lycée ou dans l'enseignement supérieur, sexe de l'élève et rang de naissance. On observe bien que l'effet propre de l'appartenance à la population étrangère ou issue de l'immigration se transforme avec l'avancement dans la scolarité. 
dans l'hexagone ou encore les élèves qui possèdent deux ou trois attributs étrangers. Quant à la transformation d'un effet négatif en effet positif, elle caractérise nettement les enfants nés à l'étranger, notamment ceux de nationalité étrangère et de parents non francophones, de même que les possesseurs de quatre attributs étrangers. Ces trois types de variation ont en commun d'être compatibles avec l'hypothèse de l'assimilation scolaire. Certes, dans quelques cas parmi lesquels figurent les enfants asiatiques, les coefficients restent stables de l'école au collège, mais l'évolution qui invaliderait l'hypothèse d'assimilation, celle de l'accentuation d'un effet négatif, n'est jamais observée. Au contraire, la tendance générale est celle d'une influence de l'appartenance aux minorités, nulle ou négative tout d'abord, mais positive ensuite (6). Observées sur les mêmes élèves, la réduction de l'inégalité de réussite des parcours entre l'école et le collège comme la transformation temporelle de l'effet associé à l'appartenance aux minorités constituent donc, selon nous, une confirmation forte de l'hypothèse de l'assimilation scolaire.

\section{UN EXAMEN EMPIRIQUE DES DÉTERMINANTS DE L'ASSIMILATION SCOLAIRE}

Pour tenter d'expliquer l'amélioration des cheminements scolaires des enfants issus de l'immigration, nous tirons parti d'informations supplémentaires collectées au cours des quatre premières années d'observation du panel. Les unes ont trait aux performances des élèves mesurées à l'entrée au collège ou au terme de celui-ci. D'autres appréhendent les souhaits et demandes familiales à l'égard du système éducatif. Les dernières décrivent certaines caractéristiques de l'environnement scolaire des élèves durant leurs deux premières années au collège. Pour des raisons de simplicité, les analyses présentées ci-dessous utilisent le critère de nationalité regroupée (Français/étranger). Elles ont toutefois été confirmées en lui substituant les autres variables d'appartenance à la population étrangère ou issue de l'immigration.

\section{L'hypothèse d'une progression plus forte des performances scolaires}

A la rentrée 1989, des épreuves standardisées de français et de mathématiques ont, pour la première fois, été administrées à tous les élèves de $6^{e}$ de France. Les résultats à ces épreuves n'ont cependant été collectés que pour une fraction des élèves du panel (Vallet et Caille, 1996b) (7). Pour les autres, seules sont disponibles les appréciations fournies au même moment par les principaux de collège sur le niveau atteint en lecture, en français écrit, en français oral et en mathématiques. Notre mesure du niveau général des élèves à leur entrée au collège utilise la première information ou, à défaut, la seconde. II s'agit d'une variable ordinale qui correspond approximativement à une distribution en quartiles. Aucune épreuve standardisée n'a en revanche été administrée aux élèves de notre échantillon durant les quatre années suivantes. Pour ceux d'entre eux parvenus en classe de $3^{e}$ générale en trois années - c'est-à-dire sans redoublement - et qui se sont présentés au brevet en juin 1993, nous disposons toutefois du résultat (succès/échec) à cet examen ; $84,5 \%$ de ces élèves l'ont réussi.

Le tableau 4 présente l'estimation d'un modèle statistique qui analyse le succès au brevet en fonction du niveau initial des élèves, de leurs caractéristiques socio-démographiques et de leur nationalité. Soit le groupe des garçons français, vivant avec leurs deux parents, aînés de familles de deux enfants où le père, ouvrier qualifié, et la mère, inactive, sont diplômés du CAP, du BEP ou du BEPC. Pour un tel élève entré au collège dans le troisième quart de la distribution des performances, la probabilité d'obtention du brevet est estimée à $87,0 \%$. Elle culmine à $95,9 \%$ $(87,0+8,9)$ si son niveau initial le situait dans le quart supérieur, mais chute à $54,1 \%(87,0-32,9)$ en cas d'appartenance au quart inférieur. Les chances d'obtention du brevet dépendent donc fortement des acquis à l'entrée au collège. De toutes les variables considérées, c'est d'ailleurs le niveau initial qui s'avère la plus influente, comme l'indiquent les coefficients estimés.

À niveau initial contrôlé, la probabilité de réussite au brevet varie aussi selon les caractéristiques socio-démographiques des élèves. Elle est significativement plus élevée chez les enfants d'agriculteur, de profession intermédiaire, de cadre ou chef d'entreprise et d'ouvrier non qualifié, suggérant ainsi que, dans leurs performances scolaires, ces élèves ont en moyenne davantage progressé que les enfants d'ouvrier qualifié de niveau initial identique. De même, dans le cadre du collège, les acquisitions des filles seraient supérieures à celles des garçons. La progression des performances serait affectée positivement par le niveau de 
Tableau 4 - Régression logistique sur l'hypothèse de progression plus forte des performances scolaires (variable dépendante : obtenir le brevet série collèges 4 ans après l'entrée en sixième)

(Accédants en $3^{e}$ générale sans redoublement candidats au brevet $-N=11190$ )

\begin{tabular}{|c|c|c|c|c|}
\hline $\begin{array}{c}\text { Modalité } \\
\text { de référence }\end{array}$ & $\begin{array}{l}\text { Modalité } \\
\text { active }\end{array}$ & Coefficient & $\begin{array}{c}\text { Test } \\
\text { statistique }\end{array}$ & $\begin{array}{c}\text { Effet } \\
\text { marginal }\end{array}$ \\
\hline Constante & & 1,90 & & $87,0 \%$ \\
\hline $\begin{array}{c}\text { PCS du chef de famille } \\
\text { ouvrier qualifié }\end{array}$ & $\begin{array}{c}\text { agriculteur } \\
\text { artisan, commerçant } \\
\text { cadre, chef d'entreprise } \\
\text { profession intermédiaire } \\
\text { employé } \\
\text { ouvrier non qualifié } \\
\text { inactif }\end{array}$ & $\begin{array}{l}0,92 \\
0,02 \\
0,34 \\
0,43 \\
0,14 \\
0,25 \\
0,10\end{array}$ & $\begin{array}{l}p<.001 \\
\text { ns } \\
p<.01 \\
p<.001 \\
\quad n s \\
p<.02 \\
\quad \text { ns }\end{array}$ & $\begin{array}{l}7,4 \% \\
3,4 \% \\
4,2 \% \\
2,5 \%\end{array}$ \\
\hline $\begin{array}{l}\text { Diplôme du père } \\
\text { cap, bep ou bepc }\end{array}$ & $\begin{array}{c}\text { sans diplôme } \\
\text { certificat d'études prim. } \\
\text { baccalauréat ou plus } \\
\text { inconnu }\end{array}$ & $\begin{array}{r}-0,24 \\
-0,10 \\
0,23 \\
-0,15\end{array}$ & $\begin{array}{c}p<.02 \\
n s \\
p<.05 \\
n s\end{array}$ & $\begin{array}{r}-3,0 \% \\
2,4 \%\end{array}$ \\
\hline $\begin{array}{l}\text { Diplôme de la mère } \\
\text { cap, bep ou bepc }\end{array}$ & $\begin{array}{c}\text { sans diplôme } \\
\text { certificat d'études prim. } \\
\text { baccalauréat ou plus } \\
\text { inconnu }\end{array}$ & $\begin{array}{r}-0,19 \\
-0,31 \\
0,40 \\
-0,39\end{array}$ & $\begin{array}{l}p<.10 \\
p<.001 \\
p<.001 \\
p<.001\end{array}$ & $\begin{array}{r}-2,3 \% \\
-4,0 \% \\
3,9 \% \\
-5,0 \%\end{array}$ \\
\hline $\begin{array}{l}\text { Activité de la mère } \\
\text { mère inactive }\end{array}$ & mère active & $-0,14$ & $p<.05$ & $-1,6 \%$ \\
\hline $\begin{array}{l}\text { sexe } \\
\text { garçon }\end{array}$ & fille & 0,29 & $p<.001$ & $2,9 \%$ \\
\hline $\begin{array}{l}\text { Taille de la famille } \\
\text { deux enfants }\end{array}$ & $\begin{array}{c}\text { un enfant } \\
\text { trois enfants } \\
\text { quatre enfants } \\
\text { cinq à sept enfants } \\
\text { huit enfants et plus }\end{array}$ & $\begin{array}{l}-0,09 \\
-0,10 \\
-0,24 \\
-0,37 \\
-0,12\end{array}$ & $\begin{array}{c}\text { ns } \\
\text { ns } \\
p<\text { <.05 } \\
p<.01 \\
\text { ns }\end{array}$ & $\begin{array}{l}-3,0 \% \\
-4,8 \%\end{array}$ \\
\hline $\begin{array}{l}\text { Rang dans la fratrie } \\
\text { rang } 1\end{array}$ & $\begin{array}{c}\text { rang } 2 \\
\text { rang } 3 \\
\text { rang } 4 \text { et plus }\end{array}$ & $\begin{array}{l}-0,17 \\
-0,05 \\
-0,21\end{array}$ & $\begin{array}{l}p<.05 \\
n s \\
p<.10\end{array}$ & $\begin{array}{l}-2,0 \% \\
-2,5 \%\end{array}$ \\
\hline $\begin{array}{c}\text { Frère ou sœur au lycée } \\
\text { ou dans l'enseignement } \\
\text { supérieur } \\
\text { non }\end{array}$ & oui & 0,35 & $p<.001$ & $3,5 \%$ \\
\hline $\begin{array}{c}\text { Structure de la famille } \\
\text { biparentale }\end{array}$ & $\begin{array}{l}\text { monoparentale } \\
\text { autre situation }\end{array}$ & $\begin{array}{l}-0,35 \\
-0,53\end{array}$ & $\begin{array}{l}p<.01 \\
p<.01\end{array}$ & $\begin{array}{l}-4,5 \% \\
-7,2 \%\end{array}$ \\
\hline $\begin{array}{l}\text { Niveau à l'entrée } \\
\text { au collège } \\
\text { troisième quart }\end{array}$ & $\begin{array}{c}\text { quart inférieur (premier) } \\
\text { second quart } \\
\text { quart supérieur }\end{array}$ & $\begin{array}{r}-1,74 \\
-0,80 \\
1,26\end{array}$ & $\begin{array}{l}p<.001 \\
p<.001 \\
p<.001\end{array}$ & $\begin{array}{r}-32,9 \% \\
-11,9 \% \\
8,9 \%\end{array}$ \\
\hline $\begin{array}{c}\text { Nationalité de l'élève } \\
\text { français }\end{array}$ & étranger & $-0,12$ & $\mathrm{~ns}$ & \\
\hline
\end{tabular}

Lecture : Parmi les élèves qui ont accédé en $3^{e}$ générale sans redoublement et se sont présentés au brevet, la probabilité d'obtenir cet examen ne diffère pas significativement entre les élèves étrangers et leurs condisciples français dont la situation familiale et sociale et le niveau à l'entrée en $6^{\mathrm{e}}$ sont semblables. Le coefficient estimé pour les élèves étrangers vaut - 0,12 , mais ne diffère pas significativement de 0 . 
diplôme de chaque parent et par la présence d'un aîné engagé dans des études longues. Elle dépendrait en revanche négativement de l'appartenance à une famille nombreuse ou monoparentale et, plus faiblement, de l'activité professionnelle maternelle. Enfin, toutes choses égales par ailleurs, la probabilité de réussite au brevet n'apparaît pas supérieure, chez les jeunes étrangers, à ce qu'elle est chez les Français ; la tendance est de sens inverse, mais s'avère très modeste et non significative.

$\grave{A}$ situation familiale et sociale et niveau initial comparables, ne se manifeste donc pas un succès plus marqué au brevet qui validerait l'hypothèse d'une meilleure progression des enfants étrangers. Notre test de cette hypothèse a toutefois pour limite d'utiliser des mesures du niveau initial et final des élèves moins précises que ne le seraient les scores obtenus à des épreuves d'évaluation standardisées.

\section{L'hypothèse du rôle des aspirations familiales}

Dans l'enquête adressée aux familles au printemps 1991 figurait cette question: "Jusqu'à quel âge souhaitez-vous que votre enfant poursuive ses études ?". Des trois réponses proposées -16 ans, 18 ans, 20 ans et plus -, $77,5 \%$ des familles de l'échantillon ont choisi la dernière, c'est-à-dire la plus ambitieuse. Le tableau 5 analyse la probabilité d'expression d'un tel souhait d'études longues à l'aide d'un modèle qui incorpore la situation familiale et sociale de l'élève, sa nationalité et deux caractéristiques scolaires.

Le niveau général et l'âge de l'enfant à son entrée au collège constituent deux signaux à partir desquels les parents ajustent leurs attentes: toutes choses égales par ailleurs, des performances plus faibles en début de $6^{e}$ comme un âge supérieur à l'âge normal rendent moins fréquent le souhait que le fils ou la fille accomplisse des études longues. Exprimer cette ambition d'une scolarité prolongée dépend fortement de la position occupée dans la structure sociale. Le diplôme de chaque parent joue dans le sens attendu. $\dot{A}$ propos de la catégorie socioprofessionnelle du chef de famille, on relève la situation particulière des agriculteurs exploitants, à l'extrémité inférieure du continuum et nettement en dessous des ouvriers comme des inactifs. Le désir d'études poussées est formulé un peu plus fréquemment pour les filles qu'il ne l'est pour les garçons. Les parents l'expriment aussi plus souvent quand, à l'intérieur de la famille, un aîné est déjà parvenu au lycée ou à l'université. Toutes choses égales par ailleurs, l'ambition que l'enfant accomplisse de longues études est légèrement plus fréquente quand la mère exerce une activité professionnelle plutôt que de rester au foyer et cette aspiration est aussi plus aiguë lorsqu'elle est exprimée par un parent qui supporte seul la charge de l'éducation.

Soit le groupe d'élèves choisi comme référence pour illustrer les enseignements de l'analyse statistique: aînés de familles de deux enfants, entrés en $6^{\theta}$ "à l'heure " et dans le troisième quart de la distribution des performances, fils d'un ouvrier qualifié et d'une femme inactive, tous deux diplômés du CAP, du BEP ou du BEPC. Pour un tel élève de nationalité française, les données conduisent à estimer à $78,5 \%$ les chances que ses parents expriment le souhait d'une poursuite d'études jusqu'à 20 ans et plus ; elles s'élèvent à $91,3 \%$ si l'enfant - et donc ses parents également - est de nationalité étrangère. Ainsi, toutes choses égales par ailleurs, que l'élève ne soit pas ressortissant français va de pair avec une ambition familiale accrue en matière de durée des études. Cet effet est quantitativement important: le coefficient estimé vaut $+1,05$. II traduit donc une différence de même ampleur que celle qui sépare les deux extrémités de l'échelle socioprofessionnelle, cadres et chefs d'entreprise d'un côté, agriculteurs exploitants de l'autre $(1,09)$. Reproduire l'analyse du tableau 5 pour les garçons d'une part et les filles d'autre part met au jour, dans les deux cas, un effet positif marqué de la nationalité étrangère, bien qu'il soit supérieur chez les premiers $(+1,25)$ à ce qu'il est chez les secondes $(+0,86)$. Enfin, des analyses complémentaires non présentées ici confirment cet effet positif quand la nationalité regroupée est remplacée par chacune des autres variables d'appartenance à la population étrangère ou issue de l'immigration. On y observe notamment que l'aspiration à des études longues culmine dans les familles d'un pays du Maghreb ou du reste de l'Afrique; d'intensité intermédiaire parmi les ressortissants d'un pays d'Asie du Sud-Est, elle est plus faible chez les familles turques et surtout celles d'Europe du Sud - portugaises essentiellement - bien qu'elle reste encore supérieure à l'ambition scolaire exprimée par les parents d'élèves français de mêmes caractéristiques. 
Tableau 5 - Régression logistique I sur l'hypothèse du rôle des aspirations familiales (variable dépendante : la famille souhaite que l'enfant poursuive ses études jusqu'à 20 ans et plus)

$(N=18538)$

\begin{tabular}{|c|c|c|c|c|}
\hline $\begin{array}{l}\text { Modalité } \\
\text { de référence }\end{array}$ & $\begin{array}{l}\text { Modalité } \\
\text { active }\end{array}$ & Coefficient & $\begin{array}{c}\text { Test } \\
\text { statistique }\end{array}$ & $\begin{array}{c}\text { Effet } \\
\text { marginal }\end{array}$ \\
\hline Constante & & 1,30 & & $78,5 \%$ \\
\hline $\begin{array}{l}\text { PCS du chef de famille } \\
\text { ouvrier qualifié }\end{array}$ & $\begin{array}{c}\text { agriculteur } \\
\text { artisan, commerçant } \\
\text { cadre, chef d'entreprise } \\
\text { profession intermédiaire } \\
\text { employé } \\
\text { ouvrier non qualifié } \\
\text { inactif }\end{array}$ & $\begin{array}{r}-0,37 \\
-0,03 \\
0,72 \\
0,53 \\
0,16 \\
-0,09 \\
-0,07\end{array}$ & $\begin{array}{c}p<.001 \\
\text { ns } \\
p<.001 \\
p<.001 \\
p<.02 \\
\text { ns } \\
\text { ns }\end{array}$ & $\begin{array}{r}-6,9 \% \\
9,7 \% \\
7,7 \% \\
2,5 \%\end{array}$ \\
\hline $\begin{array}{l}\text { Diplôme du père } \\
\text { cap, bep ou bepc }\end{array}$ & $\begin{array}{c}\text { sans diplôme } \\
\text { certificat d'études prim. } \\
\text { baccalauréat ou plus } \\
\text { inconnu }\end{array}$ & $\begin{array}{r}-0,21 \\
-0,26 \\
0,44 \\
-0,30\end{array}$ & $\begin{array}{l}p<.01 \\
p<.001 \\
p<.001 \\
p<.001\end{array}$ & $\begin{array}{r}-3,8 \% \\
-4,7 \% \\
6,6 \% \\
-5,4 \%\end{array}$ \\
\hline $\begin{array}{l}\text { Diplôme de la mère } \\
\text { cap, bep ou bepc }\end{array}$ & $\begin{array}{c}\text { sans diplôme } \\
\text { certificat d'études prim. } \\
\text { baccalauréat ou plus } \\
\text { inconnu }\end{array}$ & $\begin{array}{r}-0,28 \\
-0,33 \\
0,60 \\
-0,32 \\
\end{array}$ & $\begin{array}{l}p<.001 \\
p<.001 \\
p<.001 \\
p<.001\end{array}$ & $\begin{array}{r}-5,1 \% \\
-6,1 \% \\
8,4 \% \\
-5,9 \% \\
\end{array}$ \\
\hline $\begin{array}{l}\text { Activité de la mère } \\
\text { mère inactive }\end{array}$ & mère active & 0,15 & $p<.001$ & $2,5 \%$ \\
\hline $\begin{array}{l}\text { Sexe } \\
\text { garçon }\end{array}$ & fille & 0,24 & $p<.001$ & $3,8 \%$ \\
\hline $\begin{array}{l}\text { Taille de la famille } \\
\text { deux enfants }\end{array}$ & $\begin{array}{c}\text { un enfant } \\
\text { trois enfants } \\
\text { quatre enfants } \\
\text { cinq à sept enfants } \\
\text { huit enfants et plus }\end{array}$ & $\begin{array}{r}0,16 \\
-0,06 \\
0,00 \\
0,16 \\
0,27\end{array}$ & $\begin{array}{c}p<.05 \\
\text { ns } \\
\text { ns } \\
p<.10 \\
n s\end{array}$ & $\begin{array}{l}2,6 \% \\
2,6 \%\end{array}$ \\
\hline $\begin{array}{l}\text { Rang dans la fratrie } \\
\text { rang } 1\end{array}$ & $\begin{array}{c}\text { rang } 2 \\
\text { rang } 3 \\
\text { rang } 4 \text { et plus }\end{array}$ & $\begin{array}{r}-0,01 \\
0,13 \\
0,14\end{array}$ & $\begin{array}{c}\text { ns } \\
p<.10 \\
p<.10\end{array}$ & $\begin{array}{l}2,0 \% \\
2,3 \%\end{array}$ \\
\hline $\begin{array}{c}\text { Frère ou sœur au lycée } \\
\text { ou dans l'enseignement } \\
\text { supérieur } \\
\text { non }\end{array}$ & oui & 0,51 & $p<.001$ & $7,4 \%$ \\
\hline $\begin{array}{c}\text { Structure de la famille } \\
\text { biparentale }\end{array}$ & $\begin{array}{l}\text { monoparentale } \\
\text { autre situation }\end{array}$ & $\begin{array}{r}0,31 \\
-0,30\end{array}$ & $\begin{array}{l}p<.001 \\
p<.01\end{array}$ & $\begin{array}{r}4,8 \% \\
-5,5 \%\end{array}$ \\
\hline $\begin{array}{l}\text { Niveau à l'entrée } \\
\text { au collège } \\
\text { troisième quart }\end{array}$ & $\begin{array}{c}\text { quart inférieur (premier) } \\
\text { second quart } \\
\text { quart supérieur }\end{array}$ & $\begin{array}{r}-0,83 \\
-0,40 \\
0,47\end{array}$ & $\begin{array}{l}p<.001 \\
p<.001 \\
p<.001\end{array}$ & $\begin{array}{r}-17,1 \% \\
-7,5 \% \\
6,9 \%\end{array}$ \\
\hline $\begin{array}{l}\text { Âge d'entrée en } 6^{\mathrm{e}} \\
11 \text { ans et moins }\end{array}$ & $\begin{array}{c}12 \text { ans } \\
13 \text { ans et plus }\end{array}$ & $\begin{array}{l}-0,67 \\
-0,99\end{array}$ & $\begin{array}{l}\mathrm{p}<.001 \\
\mathrm{p}<.001\end{array}$ & $\begin{array}{l}-13,5 \% \\
-21,1 \%\end{array}$ \\
\hline $\begin{array}{c}\text { Nationalité de l'élève } \\
\text { français }\end{array}$ & étranger & 1,05 & $p<.001$ & $12,8 \%$ \\
\hline
\end{tabular}

Lecture : Toutes choses égales par ailleurs, les parents étrangers sont plus nombreux que les parents des élèves français à souhaiter que leur enfant poursuive ses études jusqu'à 20 ans et plus car le coefficient estimé est positif $(+1,05)$ et hautement significatif $(p<.001)$. À la situation de référence (décrite dans la colonne de gauche), la différence est estimée à 12,8 points. 
Lorsque leur enfant est parvenu en classe de $3^{\theta}$ générale trois ans après son entrée dans le second degré, les familles ont dû indiquer, au printemps 1993, leur souhait d'orientation au sortir du collège. Pour $79,4 \%$ des élèves concernés, les parents ont demandé en premier lieu une orientation en classe de seconde générale ou technologique. On peut alors se poser cette question: les familles issues de l'immigration qui aspiraient, plus souvent que les autres familles de même condition sociale, à ce que leur enfant entreprenne de longues études, ont-elles exprimé ce type de vœu plus fréquemment?

Tel est bien le cas (tableau 6). Le souhait d'une orientation en classe de seconde dépend principalement du niveau qu'atteignait l'élève lors de son entrée au collège, mais il est aussi sensible aux caractéristiques familiales. Ces variations ne peuvent toutefois être interprétées comme une mesure rigoureuse des inégalités de demande. Elles en donnent plutôt une vision approchée puisque les performances en fin de classe de $3^{\mathrm{e}}$ ne figurent pas dans le modèle d'analyse et que les différents groupes d'élèves ont ainsi pu connaître des progressions inégales. Dans notre perspective toutefois, le fait important est qu'entre deux élèves, l'un étranger, l'autre français, qui partagent la même situation familiale et sociale, les mêmes performances à l'entrée en $6^{\mathrm{e}}$ et l'absence de redoublement au collège, la demande parentale d'une orientation en seconde est plus probable pour le premier. Cette différence est sensible. Elle avoisine les écarts relatifs à la catégorie sociale d'appartenance et au diplôme de chaque parent et excède ceux mis au jour pour le sexe de l'élève comme pour la présence d'un aîné au lycée ou à l'université. De nouveau, cette demande plus fréquente d'une orientation en seconde a valeur presque générale : elle réapparaît en effet pour toutes les variables d'appartenance, à l'exception du nombre d'années scolaires hors de France.

Qu'à égalité de performances de leur enfant et à condition sociale identique, les familles issues de l'immigration aspirent plus souvent que les autres à de longues études et expriment plus fréquemment la demande d'orientation correspondante corrobore nettement l'hypothèse du rôle central de facteurs socio-psychologiques dans l'assimilation scolaire des enfants des minorités.

\section{La formation de l'évaluation professorale dans la procédure d'orientation}

Pour les élèves de l'échantillon parvenus sans redoublement en classe de $3^{e}$ générale, l'expression du vœu familial a été suivie de la formulation d'une proposition d'orientation par le conseil de classe : l'établissement scolaire a ou n'a pas préconisé la poursuite d'études en seconde générale ou technologique, c'est-à-dire dans la voie conduisant au baccalauréat. Le tableau 7 analyse les déterminants de cette proposition à l'aide d'un modèle où figurent le vœu de la famille, les caractéristiques socio-démographiques de l'élève, sa nationalité, son niveau général à l'entrée en $6^{e}$ et le résultat qu'il a obtenu au brevet (utilisé ici comme approximation des performances atteintes à la fin du collège).

II apparaît clairement que l'établissement scolaire est sensible à la demande familiale car l'effet du vœu d'orientation sur la proposition du conseil de classe est très prononcé. Cette dernière dépend aussi des résultats scolaires de l'élève qui se reflètent dans le succès ou l'échec au brevet comme dans le niveau général à l'entrée en $6^{e}$. Une fois ces effets "demande" et "performances" pris en compte, seuls quatre coefficients relatifs aux caractéristiques sociodémographiques sont significatifs; ils traduiraient, pour l'orientation en classe de seconde, un léger avantage des enfants de cadre ou chef d'entreprise, des élèves dont la mère est au moins bachelière, mais aussi des jeunes pour qui elle ne possède aucun diplôme ou dont le père est titulaire du CEP. Aucun des paramètres restants n'est significatif. C'est en particulier le cas de celui relatif aux enfants étrangers, virtuellement nul de surcroît $(+0,03)$. Ainsi, à demande familiale et performances scolaires identiques, la proposition formulée par le conseil de classe s'avère indépendante de l'appartenance nationale des élèves.

L'analyse présentée dans le tableau 7 n'apporte donc aucun support empirique à la thèse selon laquelle l'appartenance aux minorités serait un élément pris en compte, positivement ou négativement, dans la formation des évaluations que portent les professeurs, au cours des procédures d'orientation notamment. On peut tirer, des données recueillies à l'entrée en classe de $6^{e}$, une confirmation de cette conclusion. Elle consiste à confronter les appréciations des 
Tableau 6 - Régression logistique II sur l'hypothèse du rôle des aspirations familiales

(variable dépendante : la famille exprime un premier vœu d'orientation

en seconde générale ou technologique)

(Accédants en $3^{e}$ générale sans redoublement $-\mathrm{N}=12$ 402)

\begin{tabular}{|c|c|c|c|c|}
\hline $\begin{array}{l}\text { Modalité } \\
\text { de référence }\end{array}$ & $\begin{array}{l}\text { Modalité } \\
\text { active }\end{array}$ & Coefficient & $\begin{array}{c}\text { Test } \\
\text { statistique }\end{array}$ & $\begin{array}{c}\text { Effet } \\
\text { marginal }\end{array}$ \\
\hline Constante & & 1,22 & & $77,2 \%$ \\
\hline $\begin{array}{l}\text { PCS du chef de famille } \\
\text { ouvrier qualifié }\end{array}$ & $\begin{array}{c}\text { agriculteur } \\
\text { artisan, commerçant } \\
\text { cadre, chef d'entreprise } \\
\text { profession intermédiaire } \\
\text { employé } \\
\text { ouvrier non qualifié } \\
\text { inactif }\end{array}$ & $\begin{array}{r}0,34 \\
-0,05 \\
0,65 \\
0,46 \\
0,23 \\
0,02 \\
-0,18\end{array}$ & $\begin{array}{l}p<.02 \\
\quad \text { ns } \\
p<.001 \\
p<.001 \\
p<.01 \\
\quad \text { ns } \\
\quad \text { ns }\end{array}$ & $\begin{array}{l}5,4 \% \\
9,4 \% \\
7,1 \% \\
3,8 \%\end{array}$ \\
\hline $\begin{array}{l}\text { Diplôme du père } \\
\text { cap, bep ou bepc }\end{array}$ & $\begin{array}{c}\text { sans diplôme } \\
\text { certificat d'études prim. } \\
\text { baccalauréat ou plus } \\
\text { inconnu }\end{array}$ & $\begin{array}{r}-0,32 \\
-0,18 \\
0,37 \\
-0,17\end{array}$ & $\begin{array}{l}p<.001 \\
p<.02 \\
p<.001 \\
p<.05\end{array}$ & $\begin{array}{r}-6,0 \% \\
-3,4 \% \\
5,9 \% \\
-3,1 \%\end{array}$ \\
\hline $\begin{array}{l}\text { Diplôme de la mère } \\
\text { cap, bep ou bepc }\end{array}$ & $\begin{array}{c}\text { sans diplôme } \\
\text { certificat d'études prim. } \\
\text { baccalauréat ou plus } \\
\text { inconnu }\end{array}$ & $\begin{array}{r}-0,37 \\
-0,30 \\
0,41 \\
-0,25\end{array}$ & $\begin{array}{l}p<.001 \\
p<.001 \\
p<.001 \\
p<.01\end{array}$ & $\begin{array}{r}-7,2 \% \\
-5,7 \% \\
6,4 \% \\
-4,8 \%\end{array}$ \\
\hline $\begin{array}{l}\text { Activité de la mère } \\
\text { mère inactive }\end{array}$ & mère active & $-0,02$ & ns & \\
\hline $\begin{array}{l}\text { Sexe } \\
\text { garçon }\end{array}$ & fille & 0,46 & $p<.001$ & $7,1 \%$ \\
\hline $\begin{array}{l}\text { Taille de la famille } \\
\text { deux enfants }\end{array}$ & $\begin{array}{c}\text { un enfant } \\
\text { trois enfants } \\
\text { quatre enfants } \\
\text { cinq à sept enfants } \\
\text { huit enfants et plus }\end{array}$ & $\begin{array}{r}0,13 \\
-0,22 \\
-0,26 \\
-0,24 \\
0,05\end{array}$ & $\begin{array}{l}\quad \text { ns } \\
p<.001 \\
p<.01 \\
p<.05 \\
\quad \text { ns }\end{array}$ & $\begin{array}{l}-4,1 \% \\
-5,0 \% \\
-4,6 \%\end{array}$ \\
\hline $\begin{array}{l}\text { Rang dans la fratrie } \\
\text { rang } 1\end{array}$ & $\begin{array}{l}\text { rang } 2 \\
\text { rang } 3 \\
\text { rang } 4 \text { et plus }\end{array}$ & $\begin{array}{l}-0,21 \\
-0,10 \\
-0,22\end{array}$ & $\begin{array}{l}p<.001 \\
n s \\
p<.05\end{array}$ & $\begin{array}{l}-4,0 \% \\
-4,1 \%\end{array}$ \\
\hline $\begin{array}{c}\text { Frère ou sœur au lycée } \\
\text { ou dans l'enseignement } \\
\text { supérieur } \\
\text { non }\end{array}$ & oui & 0,45 & $p<.001$ & $6,9 \%$ \\
\hline $\begin{array}{c}\text { Structure de la famille } \\
\text { biparentale }\end{array}$ & $\begin{array}{l}\text { monoparentale } \\
\text { autre situation }\end{array}$ & $\begin{array}{l}-0,19 \\
-0,32\end{array}$ & $\begin{array}{l}p<.10 \\
p<.10\end{array}$ & $\begin{array}{l}-3,5 \% \\
-6,2 \%\end{array}$ \\
\hline $\begin{array}{l}\text { Niveau à l'entrée } \\
\text { au collège } \\
\text { troisième quart }\end{array}$ & $\begin{array}{c}\text { quart inférieur (premier) } \\
\text { second quart } \\
\text { quart supérieur }\end{array}$ & $\begin{array}{r}-1,50 \\
-0,68 \\
0,93\end{array}$ & $\begin{array}{l}\mathrm{p}<.001 \\
\mathrm{p}<.001 \\
\mathrm{p}<.001\end{array}$ & $\begin{array}{r}-34,1 \% \\
-14,1 \% \\
12,4 \%\end{array}$ \\
\hline $\begin{array}{c}\text { Nationalité de l'élève } \\
\text { français }\end{array}$ & étranger & 0,60 & $p<.001$ & $8,9 \%$ \\
\hline
\end{tabular}

Lecture : Parmi les élèves qui ont accédé en $3^{3}$ générale sans redoublement, l'expression d'un premier vœu d'orientation en seconde générale ou technologique est plus fréquente chez les élèves étrangers que chez leurs condisciples français dont la situation familiale et sociale et le niveau à l'entrée en $6^{8}$ sont semblables. $\dot{A}$ la situation de référence (décrite dans la colonne de gauche), la différence est estimée à 8,9 points. 
Tableau 7 - Régression logistique sur le comportement du conseil de classe en matière d'orientation (variable dépendante : les professeurs proposent l'orientation de l'élève en seconde générale ou technologique)

(Accédants en $3^{\theta}$ générale sans redoublement candidats au brevet $-N=11$ 190)

\begin{tabular}{|c|c|c|c|c|}
\hline $\begin{array}{c}\text { Modalité } \\
\text { de référence }\end{array}$ & $\begin{array}{l}\text { Modalité } \\
\text { active }\end{array}$ & Coefficient & $\begin{array}{c}\text { Test } \\
\text { statistique }\end{array}$ & $\begin{array}{c}\text { Effet } \\
\text { marginal }\end{array}$ \\
\hline Constante & & 2,28 & & $90,7 \%$ \\
\hline $\begin{array}{c}\text { PCS du chef de famille } \\
\text { ouvrier qualifié }\end{array}$ & $\begin{array}{c}\text { agriculteur } \\
\text { artisan, commerçant } \\
\text { cadre, chef d'entreprise } \\
\text { profession intermédiaire } \\
\text { employé } \\
\text { ouvrier non qualifié } \\
\text { inactif }\end{array}$ & $\begin{array}{r}0,31 \\
-0,07 \\
0,34 \\
0,09 \\
-0,13 \\
0,13 \\
0,19\end{array}$ & $\begin{array}{c}\mathrm{ns} \\
\mathrm{ns} \\
\mathrm{p}<.02 \\
\mathrm{~ns} \\
\mathrm{~ns} \\
\mathrm{~ns} \\
\mathrm{~ns}\end{array}$ & $2,5 \%$ \\
\hline $\begin{array}{l}\text { Diplôme du père } \\
\text { cap, bep ou bepc }\end{array}$ & $\begin{array}{c}\text { sans diplôme } \\
\text { certificat d'études prim. } \\
\text { baccalauréat ou plus } \\
\text { inconnu }\end{array}$ & $\begin{array}{r}-0,22 \\
0,30 \\
-0,09 \\
-0,04\end{array}$ & $\begin{array}{l}\text { ns } \\
p<.05 \\
\text { ns } \\
\text { ns }\end{array}$ & $2,2 \%$ \\
\hline $\begin{array}{l}\text { Diplôme de la mère } \\
\text { cap, bep ou bepc }\end{array}$ & $\begin{array}{c}\text { sans diplôme } \\
\text { certificat d'études prim. } \\
\text { baccalauréat ou plus } \\
\text { inconnu }\end{array}$ & $\begin{array}{r}0,25 \\
-0,06 \\
0,20 \\
0,05\end{array}$ & $\begin{array}{l}p<.10 \\
n s \\
p<.10 \\
n s\end{array}$ & $\begin{array}{l}1,9 \% \\
1,6 \%\end{array}$ \\
\hline $\begin{array}{l}\text { Activité de la mère } \\
\text { mère inactive }\end{array}$ & mère active & $-0,14$ & ns & \\
\hline $\begin{array}{l}\text { sexe } \\
\text { garçon }\end{array}$ & fille & 0,10 & ns & \\
\hline $\begin{array}{l}\text { Taille de la famille } \\
\text { deux enfants }\end{array}$ & $\begin{array}{c}\text { un enfant } \\
\text { trois enfants } \\
\text { quatre enfants } \\
\text { cinq à sept enfants } \\
\text { huit enfants et plus }\end{array}$ & $\begin{array}{r}-0,16 \\
0,04 \\
-0,05 \\
0,13 \\
0,51\end{array}$ & $\begin{array}{l}\text { ns } \\
\text { ns } \\
\text { ns } \\
\text { ns } \\
\text { ns }\end{array}$ & \\
\hline $\begin{array}{l}\text { Rang dans la fratrie } \\
\text { rang } 1\end{array}$ & $\begin{array}{c}\text { rang } 2 \\
\text { rang } 3 \\
\text { rang } 4 \text { et plus }\end{array}$ & $\begin{array}{r}-0,10 \\
0,12 \\
0,12\end{array}$ & $\begin{array}{l}\mathrm{ns} \\
\mathrm{ns} \\
\mathrm{ns}\end{array}$ & \\
\hline $\begin{array}{c}\text { Frère ou sœur au lycée } \\
\text { ou dans l'enseignement } \\
\text { supérieur } \\
\text { non }\end{array}$ & oui & 0,02 & ns & \\
\hline $\begin{array}{c}\text { Structure de la famille } \\
\text { biparentale }\end{array}$ & $\begin{array}{l}\text { monoparentale } \\
\text { autre situation }\end{array}$ & $\begin{array}{l}-0,16 \\
-0,08\end{array}$ & $\begin{array}{l}\text { ns } \\
\text { ns }\end{array}$ & \\
\hline $\begin{array}{l}\text { Niveau à l'entrée } \\
\text { au collège } \\
\text { troisième quart }\end{array}$ & $\begin{array}{c}\text { quart inférieur (premier) } \\
\text { second quart } \\
\text { quart supérieur }\end{array}$ & $\begin{array}{r}-0,85 \\
-0,52 \\
0,95\end{array}$ & $\begin{array}{l}p<.001 \\
p<.001 \\
p<.001\end{array}$ & $\begin{array}{r}-10,0 \% \\
-5,4 \% \\
5,5 \%\end{array}$ \\
\hline $\begin{array}{c}\text { Résultat au brevet } \\
\text { réussite }\end{array}$ & échec & $-3,16$ & $p<.001$ & $-61,4 \%$ \\
\hline $\begin{array}{l}\text { Vœu d'orientation } \\
\text { en seconde } \\
\text { oui }\end{array}$ & non & $-5,54$ & $p<.001$ & $-87,0 \%$ \\
\hline $\begin{array}{c}\text { Nationalité de l'élève } \\
\text { français }\end{array}$ & étranger & 0,03 & ns & \\
\hline
\end{tabular}

Lecture: Parmi les élèves qui ont accédé en $3^{e}$ générale sans redoublement et se sont présentés au brevet, la probabilité de recevoir une proposition d'orientation en seconde générale ou technologique ne diffère pas significativement entre les élèves étrangers et leurs condisciples français dont la situation familiale et sociale, le niveau à l'entrée en $6^{\mathrm{e}}$, le résultat au brevet et le voeu d'orientation formulé sont semblables. Le coefficient estimé pour les èlèves étrangers vaut $+0,03$ et ne diffère pas significativement de 0 . 
élèves fournies par l'établissement en lecture, français écrit, français oral et mathématiques aux performances des mêmes élèves mesurées objectivement par les scores aux épreuves d'évaluation standardisées. Selon cette analyse, la nationalité de l'élève n'a pas affecté le jugement porté, en début de $6^{\mathrm{e}}$, sur son niveau en français ou en mathématiques (8).

\section{L'effet du contexte de scolarisation}

Pour les élèves de notre échantillon, deux variables supplémentaires permettent de caractériser le contexte de leur scolarisation : la proportion d'enfants étrangers dans la classe fréquentée durant l'année 1989-1990, le fait que l'établissement scolaire de l'année 1990-1991 était ou n'était pas situé dans une zone d'éducation prioritaire. Le lien qu'entretiennent ces deux variables avec l'appartenance aux minorités est net. Dans l'échantillon considéré, $32,1 \%$ des élèves étrangers étaient scolarisés dans une ZEP ; ce n'était le cas que pour 9,5\% des enfants français (9). De même, $49,0 \%$ des Français n'avaient aucun condisciple étranger dans leur classe de $6^{\mathrm{e}}$, mais près de la moitié des étrangers étaient dans une classe qui comprenait un quart ou plus d'un quart d'enfants non français.

Le tableau 8 présente une analyse statistique qui vise à évaluer l'effet de ces caractéristiques contextuelles sur le cheminement scolaire des élèves. En raison de la période où elles sont observées, le succès ou l'échec du parcours au collège est appréhendé deux années après l'entrée en $6^{\mathrm{e}}$ : l'élève est ou n'est pas parvenu sans redoublement en classe de $4^{\mathrm{e}}$ générale. Pour isoler l'effet spécifiquement associé au contexte de scolarisation, niveau de performance initial et variables socio-démographiques sont simultanément introduits dans le modèle de régression.

II apparaît clairement que les caractéristiques du contexte de scolarisation ont affecté le déroulement du parcours scolaire des élèves. Néanmoins, celui-ci dépend surtout du niveau de performance initial et les variables contextuelles sont aussi moins influentes que les caractéristiques individuelles des collégiens (origine sociale, diplôme des parents et sexe notamment). En outre, l'effet du contexte de scolarisation n'est pas univoque. Bien qu'une forte présence d'enfants étrangers dans la classe de $6^{\mathrm{e}}$ paraisse affecter négativement les parcours scolaires des collégiens, cet effet, à peine significatif, est d'intensité négligeable : à la situation de référence décrite dans la colonne de gauche du tableau 8 , un accroissement de $10 \%$ de la proportion d'élèves étrangers s'accompagne d'une diminution de 0,4 point seulement de la probabilité d'accès en $4^{\mathrm{e}}$ générale sans redoublement. Relativement à des collégiens de niveau initial et caractéristiques socio-démographiques identiques, mais non scolarisés en ZEP, l'appartenance à une zone d'éducation prioritaire constitue en revanche un atout pour l'accès en deux années à la classe de $4^{e}$ générale. D'amplitude analogue à l'avantage dont disposent les enfants vivant avec leurs deux parents sur ceux de famille monoparentale, cet effet positif n'est pas négligeable et pourrait être lié aux conditions de scolarisation, politiques éducatives et actions incitatives souvent développées dans les ZEP.

Nous réservons pour un examen ultérieur la question de savoir si ces caractéristiques du contexte de scolarisation affectent différemment les cheminements des enfants des minorités et ceux des autres élèves. Dès à présent, il est toutefois possible d'établir que l'amélioration des parcours des enfants issus de l'immigration n'a pas sa source dans le contexte de scolarisation particulier que nombre d'entre eux connaissent et qu'elle paraît assez indépendante de celui-ci. Le tableau 8 montre en effet que, durant les deux premières années du collège, les enfants étrangers ont connu de meilleurs parcours que leurs condisciples français de même niveau initial, de caractéristiques socio-démographiques identiques et pour lesquels le contexte de scolarisation était semblable. Cet effet (coefficient de $+0,55$ ) apparait beaucoup plus fort que celui des deux variables d'environnement et retirer ces dernières du modèle d'analyse - c'est-àdire raisonner sans tenir compte du contexte de scolarisation - ne l'affecte que très peu: le coefficient associé à la nationalité étrangère s'élève alors à $+0,53$.

\section{**}

Dans cet article, nous avons désigné par assimilation scolaire des minorités issues de l'immigration le processus temporel par lequel, avec l'avancement dans les études, les parcours des enfants de ces minorités s'améliorent et convergent en moyenne vers ceux des autres élèves. 
Tableau 8 - Régression logistique sur l'effet du contexte de scolarisation (variable dépendante : parvenir en classe de quatrième générale sans avoir redoublé au collège)

$$
(\mathrm{N}=18538)
$$

\begin{tabular}{|c|c|c|c|c|}
\hline $\begin{array}{l}\text { Modalité } \\
\text { de référence }\end{array}$ & $\begin{array}{l}\text { Modalité } \\
\text { active }\end{array}$ & Coefficient & $\begin{array}{c}\text { Test } \\
\text { statistique }\end{array}$ & $\begin{array}{c}\text { Effet } \\
\text { marginal }\end{array}$ \\
\hline Constante & & 1,79 & & $85,7 \%$ \\
\hline $\begin{array}{l}\text { PCS du chef de famille } \\
\text { ouvrier qualifié }\end{array}$ & $\begin{array}{c}\text { agriculteur } \\
\text { artisan, commerçant } \\
\text { cadre, chef d'entreprise } \\
\text { profession intermédiaire } \\
\text { employé } \\
\text { ouvrier non qualifié } \\
\text { inactif }\end{array}$ & $\begin{array}{r}0,36 \\
0,08 \\
0,43 \\
0,28 \\
0,05 \\
-0,09 \\
0,07\end{array}$ & $\begin{array}{l}\mathrm{p}<.01 \\
\mathrm{~ns} \\
\mathrm{p}<.001 \\
\mathrm{p}<.001 \\
\mathrm{~ns} \\
\mathrm{~ns} \\
\mathrm{~ns}\end{array}$ & $\begin{array}{l}3,9 \% \\
4,5 \% \\
3,1 \%\end{array}$ \\
\hline $\begin{array}{l}\text { Diplôme du père } \\
\text { cap, bep ou bepc }\end{array}$ & $\begin{array}{c}\text { sans diplôme } \\
\text { certificat d'études prim. } \\
\text { baccalauréat ou plus } \\
\text { inconnu }\end{array}$ & $\begin{array}{r}-0,20 \\
-0,09 \\
0,24 \\
-0,11\end{array}$ & $\begin{array}{c}p<.01 \\
n s \\
p<.01 \\
n s\end{array}$ & $\begin{array}{r}-2,6 \% \\
2,7 \%\end{array}$ \\
\hline $\begin{array}{l}\text { Diplôme de la mère } \\
\text { cap, bep ou bepc }\end{array}$ & $\begin{array}{c}\text { sans diplôme } \\
\text { certificat d'études prim. } \\
\text { baccalauréat ou plus } \\
\text { inconnu }\end{array}$ & $\begin{array}{r}-0,42 \\
-0,27 \\
0,45 \\
-0,24\end{array}$ & $\begin{array}{l}p<.001 \\
p<.001 \\
p<.001 \\
p<.01\end{array}$ & $\begin{array}{r}-6,0 \% \\
-3,6 \% \\
4,7 \% \\
-3,2 \%\end{array}$ \\
\hline $\begin{array}{l}\text { Activité de la mère } \\
\text { mère inactive }\end{array}$ & mère active & $-0,07$ & ns & \\
\hline $\begin{array}{l}\text { Sexe } \\
\text { garçon }\end{array}$ & fille & 0,66 & $p<.001$ & $6,4 \%$ \\
\hline $\begin{array}{l}\text { Taille de la famille } \\
\text { deux enfants }\end{array}$ & $\begin{array}{c}\text { un enfant } \\
\text { trois enfants } \\
\text { quatre enfants } \\
\text { cinq à sept enfants } \\
\text { huit enfants et plus }\end{array}$ & $\begin{array}{r}0,06 \\
-0,04 \\
-0,20 \\
-0,11 \\
0,12\end{array}$ & $\begin{array}{c}\mathrm{ns} \\
\mathrm{ns} \\
\mathrm{p}<.01 \\
\mathrm{~ns} \\
\mathrm{~ns}\end{array}$ & $-2,6 \%$ \\
\hline $\begin{array}{l}\text { Rang dans la fratrie } \\
\text { rang } 1\end{array}$ & $\begin{array}{l}\text { rang } 2 \\
\text { rang } 3 \\
\text { rang } 4 \text { et plus }\end{array}$ & $\begin{array}{r}0,02 \\
-0,04 \\
-0,07\end{array}$ & $\begin{array}{l}\mathrm{ns} \\
\mathrm{ns} \\
\mathrm{ns}\end{array}$ & \\
\hline $\begin{array}{c}\text { Frère ou sœur au lycée } \\
\text { ou dans l'enseignement } \\
\text { supérieur } \\
\text { non }\end{array}$ & oui & 0,15 & $p<.01$ & $1,7 \%$ \\
\hline $\begin{array}{c}\text { Structure de la famille } \\
\text { biparentale }\end{array}$ & $\begin{array}{l}\text { monoparentale } \\
\text { autre situation }\end{array}$ & $\begin{array}{l}-0,26 \\
-0,30\end{array}$ & $\begin{array}{l}p<.001 \\
p<.01\end{array}$ & $\begin{array}{l}-3,5 \% \\
-4,1 \%\end{array}$ \\
\hline $\begin{array}{l}\text { Niveau à l'entrée } \\
\text { au collège } \\
\text { troisième quart }\end{array}$ & $\begin{array}{c}\text { quart inférieur (premier) } \\
\text { second quart } \\
\text { quart supérieur }\end{array}$ & $\begin{array}{r}-2,46 \\
-1,20 \\
1,80\end{array}$ & $\begin{array}{l}p<.001 \\
p<.001 \\
p<.001\end{array}$ & $\begin{array}{r}-51,9 \% \\
-21,4 \% \\
11,7 \%\end{array}$ \\
\hline $\begin{array}{l}\text { Collège en zep } \\
\text { non }\end{array}$ & oui & 0,25 & $p<.001$ & $2,8 \%$ \\
\hline $\begin{array}{l}\% \text { d'élèves étrangers } \\
\text { dans la classe de } 6^{\mathrm{e}}\end{array}$ & $\begin{array}{c}10 \% \text { d'élèves étrangers } \\
\text { en plus }\end{array}$ & $-0,03$ & $p<.10$ & $-0,4 \%$ \\
\hline $\begin{array}{c}\text { Nationalité de l'élève } \\
\text { français }\end{array}$ & étranger & 0,55 & $p<.001$ & $5,6 \%$ \\
\hline
\end{tabular}

Lecture : Les élèves étrangers ont plus souvent accédé en $4^{e}$ générale sans avoir redoublé au collège que leurs condisciples français dont la situation familiale et sociale, le niveau à l'entrée en $6^{\mathrm{e}}$ et le contexte de scolarisation sont semblables car le coefficient estimé est positif $(+0,55)$ et hautement significatif $(p<.001)$. A la situation de référence (décrite dans la colonne de gauche), la différence est estimée à 5,6 points. 
Nous avons mis en évidence un tel processus, dans la société française, en comparant la réussite des cheminements qu'ont connus les mêmes élèves, à l'école élémentaire, puis au collège. Notre examen empirique des déterminants de l'assimilation scolaire suggère qu'elle ne dépend guère des évaluations portées par les professeurs sur les enfants des minorités ou des caractéristiques contextuelles de leur scolarisation. Nous n'avons pas non plus mis en évidence que l'assimilation scolaire des enfants issus de l'immigration tenait à une progression plus forte de leurs performances. Une telle conclusion apparaît cependant provisoire car l'examen de cette hypothèse devrait être repris à l'aide de mesures des acquisitions plus précises que celles qui étaient à notre disposition. En revanche, nous avons nettement observé que les attentes et les espoirs que les familles immigrées placent dans le système éducatif sont un levier important du processus d'assimilation scolaire: les familles immigrées ont, ceteris paribus, des souhaits plus vifs en matière d'études longues et expriment, pour leurs enfants, des demandes d'orientation plus ambitieuses auxquelles les établissements sont sensibles. Ce résultat conduit à penser que, dans la société française, les familles immigrées perçoivent le système éducatif comme une voie importante de mobilité sociale. II suggère également que l'absence ou la faiblesse de la scolarisation parentale n'a pas les mêmes effets, sur la façon de considérer l'avenir des enfants, selon qu'elle est due à l'inexistence ou à la déficience du système éducatif dans le pays d'origine - ce qui est fréquemment le cas des familles immigrées - ou bien qu'elle relève de difficultés scolaires rencontrées au cours de la jeunesse ce qui concerne plus souvent les parents, de même niveau d'études, ayant toujours vécu en France.

Dans la société française, l'assimilation scolaire des élèves des minorités paraît ainsi fortement liée aux conditions institutionnelles de fonctionnement du collège unique où le point de vue des familles occupe une place importante dans les décisions d'orientation. Des recherches antérieures ont mis en évidence les biais sociaux associés à un tel fonctionnement: toutes choses égales par ailleurs, le fait de demander une orientation accroît la probabilité de l'obtenir ; or, à niveau scolaire moyen, les familles de cadres "demandent plus" que les familles ouvrières et la prise en compte de ces vœux a dès lors pour effet de creuser les inégalités de carrières scolaires entre les deux milieux sociaux (Duru et Mingat, 1985 et 1988). Sans remettre en cause ce résultat, notre recherche le complète en soulignant a contrario le rôle qu'a le fonctionnement du collège unique dans l'assimilation scolaire des élèves des minorités. La place qu'occuperaient les arrangements institutionnels dans ce processus est encore suggérée par une comparaison avec l'Allemagne où le second degré comprend trois filières hiérarchisées - Gymnasium, Realschule, Hauptschule - entre lesquelles les élèves sont orientés au sortir de l'école primaire (10 ans environ). Une recherche comparable à la nôtre y met en évidence que, par rapport aux jeunes Allemands de caractéristiques sociodémographiques identiques, les enfants italiens, turcs et, à un degré moindre, yougoslaves fréquentent plus souvent la filière de scolarisation la moins valorisée, quittent plus fréquemment cette voie du système éducatif sans y avoir obtenu de diplôme d'apprentissage et effectuent moins souvent leurs études secondaires dans un lycée (Alba, Handl et Müller, 1994). Si la durée d'exposition à l'école du pays d'accueil est un levier important de l'amélioration des parcours que connaissent les enfants issus de l'immigration, il est possible qu'une orientation précoce joue en leur défaveur. L'opposition des résultats allemands et français renverrait alors aux arrangements institutionnels distincts des deux pays : la division rapide entre filières hiérarchisées dans le système allemand opposée au collège unique de la société française.

Louis-André Vallet

LASMAS-Institut du Longitudinal (CNRS, Caen) et Laboratoire de Sociologie Quantitative (CREST, Paris) 
(1) Voir, pour une revue des études quantitatives antérieures sur les parcours, les performances et la progression des performances des élèves étrangers dans le système scolaire français, Vallet et Caille (1996a, chapitre premier).

(2) Un second indicateur de réussite utilise l'information recueillie au cours des deux premières années d'observation du panel : être parvenu en classe de $4^{e}$ générale sans avoir redoublé au collège ; $73,3 \%$ des élèves de l'échantillon étaient dans cette situation favorable. Cet indicateur est utilisé dans l'analyse de l'effet du contexte de scolarisation.

(3) Les proportions présentées sont calculées à partir de l'échantillon initial ( $N=18657$ ).

(4) Pour quelques groupes de taille réduite que le tableau 1 permet de distinguer, l'écart mesuré à l'école élémentaire ou au collège est positif, manifestant ainsi que la réussite de ces élèves est meilleure que celle du groupe de référence. Cela ne contredit pas l'hypothèse de l'assimilation scolaire. La tendance observée est en effet celle de l'accentuation d'un écart positif ou de la transformation d'un écart négatif en écart positif.

(5) Les coefficients d'un modèle de régression logistique sont les logarithmes des odds ratios estimés sous le modèle considéré. L'analyse statistique permet ainsi de conclure qu'à situation familiale et sociale identique, les chances de réussite plutôt que d'échec du parcours scolaire au collège ont été exp $(0,30)$, soit 1,3 fois plus fortes pour les jeunes étrangers par rapport aux enfants français.

(6) À l'opposé, les effets associés aux caractéristiques sociodémographiques qui décrivent la situation familiale et sociale des élèves s'avèrent beaucoup plus stables dans le temps ; on consultera sur ce point, dans Vallet et Caille (1996a), les tableaux III-2 et VI-2.

(7) Leur analyse montre que, comparativement aux entrants en $6^{e}$ français, les enfants étrangers obtenaient en moyenne des scores inférieurs de 8,7 points (sur un total de 100) en français et de 6,1 points en mathématiques. Ces écarts tiennent largement aux caractéristiques socio-démographiques des groupes comparés. À situation familiale et sociale identique, la différence n'est en effet plus significative en mathématiques et se réduit à 1,4 point en français. Des résultats de même nature ont aussi été obtenus aux Pays-Bas (Van't Hof et Dronkers, 1994).

(8) Voir, pour une présentation détaillée, Vallet et Caille (1996a, p. 135).

(9) Ou encore, parmi les élèves d'un collège en ZEP, on comptait $22,0 \%$ d'étrangers et $35,8 \%$ d'enfants présentant au moins un attribut étranger; les proportions correspondantes valaient $5,9 \%$ et $17,5 \%$ parmi les élèves qui n'appartenaient pas à une zone d'éducation prioritaire.

\section{BIBLIOGRAPHIE}

ALBA R.D., HANDL J. et MÜLLER W. (1994). - Ethnic Inequalities in the German School System, Mannheimer Zentrum für Europäische Sozialforschung (MZES), Arbeitspapier AB I, Nr. 8, Mannheim.

ALBA R. et NEE V. (1996). - How relevant is assimilation?, International Conference "Migration, Integration and Ethnic Conflicts ", Mannheim, June 19-22.

BRESSOUX P. (1994). - Les effets de la formation initiale et de l'expérience professionnelle des instituteurs. Etude portant sur le CE2. Les dossiers d'Éducation et Formations, 36, (Ministère de l'Éducation nationale, DEP).

CLIFTON R.A., PERRY R.P., PARSONSON K. et HRYNIUK S. (1986). - Effects of Ethnicity and Sex on Teachers' Expectations of Junior High School Students. Sociology of Education, 59(1), p. 58-67.

CLIFTON R.A., WILLIAMS T. et CLANCY J. (1991). The Academic Attainment of Ethnic Groups in Australia : A Social Psychological Model. Sociology of Education, 64(2), p. 111-126.

DURU M. et MINGAT A. (1985 et 1988). - De l'orientation en fin de $5^{\mathrm{e}}$ au fonctionnement du collège. 1 - Évaluation de la procédure. 2 - Progression, notation, orientation : l'impact du contexte de scolarisation. Les cahiers de I'IREDU, 42 et 45 .

DURU-BELLAT M. et JAROUSSE J.-P. (1996). - Le masculin et le féminin dans les modèles éducatifs des parents. Économie et Statistique, 293, p. 77-93.

ERNST B. et RADICA K. (1994). - Les élèves au cycle d'observation: caractéristiques, performances en français et en mathématiques, orientation en fin de cycle, p. 13-42 in "Évaluation au cycle d'observation (1989-1992). Cycle en 3 ans, redoublement et autres mesures d'aide: quelle efficacité ?". Les dossiers d'Éducation et Formations, 40, (Ministère de l'Éducation nationale, DEP).

GRISAY A. (1993). - Le fonctionnement des collèges et ses effets sur les élèves de sixième et de cinquième. Les dossiers d'Éducation et Formations, 32, (Ministère de l'Éducation nationale, DEP).

MEURET D. (1994). - L'efficacité de la politique des zones d'éducation prioritaire dans les collèges. Revue française de pédagogie, 109, p. 41-64.

MINGAT A. (1991). - Expliquer la variété des acquisitions au cours préparatoire : les rôles de l'enfant, la famille et l'école. Revue française de pédagogie, 95 , p. 47-63.

MULLER C. et KERBOW D. (1993). - Parent Involvement in the Home, School, and Community, $p$. 13-42 in Parents, Their Children, and Schools, Schneider B. and Coleman J.S. (eds.), Boulder: Westview Press.

NEIDERT L.J. et FARLEY R. (1985). - Assimilation in the United States: An Analysis of Ethnic and 
Generation Differences in Status and Achievement. American Sociological Review, 50(6), p. 840-850.

SERRA N. et THAUREL-RICHARD M. (1994). Acquisitions des élèves au CE2 et pratiques pédagogiques. Revue française de pédagogie, 107, p. 43-62.

SMITH D.J. et TOMLINSON S. (1989). - The School Effect. A Study of Multi-Racial Comprehensives. London : Policy Studies Institute.

TRIBALAT M. (1996). - De l'immigration à l'assimilation. Enquête sur les populations d'origine étrangère en France. Paris : La Découverte-INED.

VALLET L.-A. (1988). - L'évolution de l'inégalité des chances devant l'enseignement. Un point de vue de modélisation statistique. Revue française de sociologie, 29(3), p. 395-423.

VALLET L.-A, et CAILLE J.-P. (1995). - Les carrières scolaires au collège des élèves étrangers ou issus de l'immigration. Éducation et Formations, 40 , p. $5-14$.

VALLET L.-A. et CAILLE J.-P. (1996a). - Les élèves étrangers ou issus de l'immigration dans l'école et le collège français. Une étude d'ensemble. Les dossiers d'Éducation et Formations, 67, (Ministère de l'Éducation nationale, DEP).

VALLET L.-A. et CAILLE J.-P. (1996b). - Niveau en français et en mathématiques des élèves étrangers ou issus de l'immigration. Économie et Statistique, 293, p. 137-153.

VAN'T HOF L. et DRONKERS J. (1994). - Differences in Educational Attainment of Children from Various Groups of Recent Immigrants in the Netherlands: Class, Family or Migrant Culture ? Migration. A European Journal of International Migration and Ethnic Relations, 26, p. 5-48. 